\title{
Equilibrium Blocking in Large Quasilinear Economies*
}

\author{
Yusuke Kamishiro ${ }^{\dagger}$ and Roberto Serrano ${ }^{\ddagger}$
}

This version: October 2009

\begin{abstract}
We study information transmission in large interim quasilinear economies using the theory of the core. We concentrate on the core with respect to equilibrium blocking, a core notion in which information is transmitted endogenously within coalitions, as blocking can be understood as an equilibrium of a communication mechanism used by players in coalitions. We consider independent, ex-post and signal-based replicas of the basic economy. For each, we offer an array of negative and positive convergence results as a function of the complexity of the mechanisms used by coalitions. We identify conditions under which asymmetric information remains as an externality and non-market outcomes stay in the core, as well as those for the core to converge to the set of incentive compatible ex-post Walrasian allocations. Further, all the results are robust to the relaxation of the incentive constraints, and hence suggest a process through which information may get incorporated into a fully revealing equilibrium price function.

Keywords: Core w.r.t. Equilibrium Blocking, Core Convergence, Independent Replicas, Ex-Post Replicas, Signal-Based Replicas, Information Transmission, Communication Mechanisms, Mediation, Rational Expectations Equilibrium.
\end{abstract}

JEL Classification: C71, C72, D51, D82.

\footnotetext{
*Some of the results reported here circulated previously under the title "Information Transmission and Core Convergence in Quasilinear Economies." We thank Geoffroy de Clippel, Francoise Forges, Andreu Mas-Colell, Roger Myerson, Andy Postlewaite, seminar audiences at Brown, Carlos III, Hitotsubashi, Tokyo Institute of Technology and Waseda, and participants at the 2008 NSF/CEME Decentralization Conference at Tulane, the 2009 Canadian Economic Theory Conference at Toronto, and the 2009 European Workshop on General Equilibrium at Pompeu Fabra for comments and encouragement. Serrano acknowledges the hospitality of CEMFI and the hospitality and financial support of Universidad Carlos III in Madrid.

${ }^{\dagger}$ Dept. of Economics, Brown University, Providence, RI 02912, U.S.A.; yusuke_kamishiro@brown. edu

‡Dept. of Economics, Brown University, Providence, RI 02912, U.S.A., and IMDEASocial Sciences, Madrid, Spain; roberto_serrano@brown.edu
} 


\section{Introduction}

This paper studies information transmission in large economies and sheds light on how information may get incorporated into a fully revealing rational expectations equilibrium price function. To do so, we use an approach based on the core. We concentrate on the core based on endogenous information transmission. We shall call such a core the core with respect to (w.r.t.) equilibrium blocking, in that each blocking move is identified with an equilibrium of a communication mechanism used by coalitions. This was the unifying approach proposed in Serrano and Vohra (2007), and we pursue it further here. Indeed, as shown in that paper, this general notion of core encompasses other notions previously proposed in the literature: if coalitions are restricted to use deterministic mechanisms that involve only one coalition, it boils down to the credible core of Dutta and Vohra (2005); if they can use random mechanisms that are subjected to a measurability restriction explained below, the approach yields Myerson's (2007) virtual utility core; and if such restrictions in random mechanisms are removed, one obtains the randomized mediated core of Serrano and Vohra (2007). ${ }^{1}$

The core convergence theorem is an important cornerstone of the relationship between the predictions of game theory in large economies and competitive equilibrium allocations. Many results have followed the seminal works of Debreu and Scarf (1963) and Aumann (1964); see Anderson (2008) for a recent survey. This paper focuses on interim economies with hidden types, a leading class of problems within asymmetric information (in it, each agent has private information that is not public knowledge). As already stated, we shall concentrate on a core concept that models the information transmission that goes on within each coalition endogenously. We shall present an array of negative and positive results for the main replica processes proposed in earlier literature (independent, ex-post and signal-based), leading to a fairly comprehensive picture of the problem at the interim stage.

\footnotetext{
${ }^{1}$ The literature has been asking the question of which is the "right" notion of asymmetric information core for some time. In this respect, we think that understanding our results as "this core converges" and "that core does not converge" is not the best reading of our contribution. As already stated, several core concepts in the literature are all illustrations of the same core, one in which the tools of mechanism design are used to set up each blocking move as a Bayesian equilibrium of a communication mechanism among the participants. Also, to avoid the long name of the concept, we shall shorten it: for example, instead of saying "core w.r.t. equilibrium blocking of deterministic mechanisms," we shall say from now on "core of deterministic mechanisms," and so on.
} 
The core convergence question in the ex-ante stage (i.e., that in which no agent has received any private information) has also been studied. For this stage, if no restrictions are imposed on the set of allocations, the question is simply a reformulation of the standard problem in an Arrow-Debreu economy framework. Indeed, the most interesting cases happen when either measurability or incentive compatibility restrictions are imposed. ${ }^{2}$

There are also results obtained for the ex-post stage (i.e., that in which all private information has been made common knowledge). ${ }^{3}$ The results in the current paper will connect with some of these, due to the information transmission embodied in the interim core we study, which, if one allows unrestricted communication blocking mechanisms, places it closer to an expost solution concept.

For the interim stage, our concern here, we shall consider independent replicas, ex-post replicas and signal-based replicas. Independent replicas were introduced in Gul and Postlewaite (1992) and also studied in Forges, Heifetz and Minelli (2001). Independent replicas also replicate the states of the world, and in them, each agent's preferences depend only on the information pertinent to his replica. Thus, agents' information is relevant to a negligible set of agents in large economies. Serrano, Vohra and Volij (2001) studies ex-post replicas, i.e., replicas in which the set of states of the world is not replicated with the economy. This implies that, already in the second replica, the economy is one of non-exclusive information in the sense of Postlewaite and Schmeidler (1986) and incentive constraints become redundant. The signal-based replicas of McLean and Postlewaite (2005) lie somewhere in between: the set of underlying unobservable states is not replicated, and agents receive signals that, if pooled, come close to identifying the true state, although this never happens. The unobservable state is a common value in agents' preferences, while types -signals- are purely informational, affecting

\footnotetext{
${ }^{2}$ Forges, Heifetz and Minelli (2001) study the convergence of the ex-ante incentive compatible core using the independent replication process. McLean and Postlewaite (2005) establish a positive convergence result for the ex-ante incentive compatible core in a signalbased replication process. The reader is referred to Allen and Yannelis (2001) and Forges, Minelli and Vohra (2002) for general surveys of the area.

${ }^{3}$ See, for example, Einy, Moreno and Shitovitz (2000a), who work with atomless economies, assuming the number of states is finite. Although we shall argue that in expost replicas the continuum case is covered, it is not clear to us how to extend our other replicas analysis to atomless economies: the state space would also become "atomless," and then, it would be difficult to define the join information of a coalition, for example, hindering the use of the arguments in that paper.
} 
only probability assessments. Agents in the three replica processes become informationally small in different ways and it is important to understand the different properties of information flows in each case.

Our starting point is to consider economies in which the state is not verifiable ex-post, and thus, incentive constraints become important. For these economies, one can use the tools of mechanism design to propose a core notion in which communication within coalitions is endogenous; this notion of "core w.r.t. equilibrium blocking" takes center stage in our study and we describe it in the sequel. However, importantly, we shall also study how our main results extend to economies without incentive constraints. ${ }^{4}$

We shall assume that agents in our economies have quasilinear preferences. For this class, different core existence results have been provided (see Dutta and Vohra (2005), Myerson (2007)). It is important to stress that nonemptiness is in general hard to achieve for the interim core once incentive constraints are in place, as pointed out by Vohra (1999) and Forges, Mertens and Vohra (2002). Aside non-emptiness, some of our proofs also rely on the quasilinearity assumption (especially the generalized version of the first welfare theorem, i.e., the inclusion of equilibrium in the core). Myerson (2007) notes that, even in quasilinear settings, random blockings make a difference to the definition of the core in the presence of asymmetric information (unlike what is known for complete information games). The current work shows that, even in quasilinear economies, depending on the replica process and on how much information transmission one allows in random blocking plans, the answer that one obtains to the core convergence question is also very different.

If blocking communication mechanisms are deterministic and use only one coalition, our core yields the credible core. The credible core, proposed in Dutta and Vohra (2005), is the set of incentive compatible allocations immune to credible objections. A coalition has a credible objection if it can identify an informational event such that the types of agents involved in the event are the only ones that prefer the alternative proposed to the status quo, given that the other types behave as prescribed in the objection. This

\footnotetext{
${ }^{4} \mathrm{~A}$ word about the proof method followed here is called for. The core we employ relies heavily on the machinery of mechanism design and the revelation principle. Thus, incentive constraints are at the center of the analysis. These cause problems to non-emptiness of the core. We are careful in our proofs to construct arguments that rely on information transmission, with independence of whether or not it incorporates incentive constraints. The scope of our conclusions is enhanced as a result.
} 
self-selection ensures that a type wishes to participate in the objection if and only if it is consistent with the objection's event. Therefore, the information transmitted in the objection via the event is "credible" in that the types that are not part of it have no incentive to join the objection; hence the name of the concept. But there is no good reason to restrict attention to such communication mechanisms, the main point being that blocking is to be understood as an equilibrium phenomenon from which information transmission is derived endogenously. For instance, if a certain class of measurable random communication mechanisms is permitted, our core yields the concept proposed in Myerson (2007). Based on the virtual utility construct, Myerson (2007) proposes a core notion that imposes the credibility requirements over random coalition formation and random allocations for each coalition. Serrano and Vohra (2007), which sets up the Dutta-Vohra and Myerson objections as communication mechanisms played by the agents in each coalition and derives their objections' inequalities from the equilibria of such communication games, also relax the measurability restriction used in Myerson's mechanisms. Indeed, the use of unrestricted random blockings is one difference between Myerson's (2007) concept and that arrived at in Serrano and Vohra (2007), which had not been previously emphasized, and such a difference plays an important role in some of our results. The issue concerns the possibility of information transmission within a random blocking plan across the different coalitions that comprise it. Correspondingly, we shall talk about the core of an increasingly large class of communication mechanisms: deterministic mechanisms (the credible core of Dutta-Vohra), measurable random mechanisms (the virtual utility core of Myerson), and unrestricted random mechanisms (the randomized mediated core of Serrano-Vohra). Details will be provided in Section 2 .

Our results show both negative and positive convergence conclusions, and both are important in the understanding of information flows in large economies. First, in independent replicas, we shall propose a simple robust example to demonstrate that the core of measurable random mechanisms -and thus, of deterministic mechanisms a fortiori- does not shrink to any price equilibrium notion. However, if information transmission is allowed to flow within the coalitions that participate in a given random blocking plan, the strictly incentive compatible allocations in the core of unrestricted mechanisms converge to the set of incentive compatible ex-post Walrasian allocations. We remark again that incentive constraints are not of the essence.

In ex-post replicas, Serrano, Vohra and Volij (2001) have exhibited a 
robust failure of the core convergence theorem for cores in which there is only exogenous information transmission. Essentially, for cores that use a limited amount of exogenously specified information transmission (e.g., the coarse core of Wilson (1978)), the core is "too large" and does not shrink down to the set of price allocations; and the core that exogenously allows any kind of communication (Wilson's fine core) is often "too small," even empty, still when price allocations can be supported. In contrast, we shall show that the core of deterministic mechanisms converges to the set of expost Walrasian allocations (recall that in these replicas incentive constraints can be dropped in the second replica). Thus, in this case, there is no need to resort to random communication mechanisms to obtain a full revelation of information result; modelling communication as an equilibrium phenomenon within a coalition suffices.

In signal-based replicas, we show a probabilistic convergence result for the core of unrestricted mechanisms. Specifically, for large enough replicas the probability of a specific informational event is arbitrarily close to 1 . The informational event in question consists of those signal profiles for which at any core allocation the ex-post utility of almost all agents is arbitrarily close to that obtained at an ex-post Walrasian allocation in some state. For these replicas, we do not know the answer to the convergence question for the core of restricted mechanisms (deterministic, for instance).

Regarding the quasilinearity assumption, we note that our core shrinking argument in independent and ex-post replicas does not rely on it. That is, even without it, one can show that if such a replica of an allocation is in the core in all replicas, it will have the ex-post Walrasian property. In contrast, the probabilistic core shrinking in signal-based replicas does rely on quasilinearity. And more importantly, what definitely fails in the absence of quasilinearity is the other part of the argument, that is, the inclusion of equilibrium in the core, since insurance opportunities exist that render the equilibrium allocation interim inefficient (Laffont (1985)).

To conclude with the introduction, our findings shed light on the rather more mysterious information transmission that goes on within a price function in a fully revealing rational expectations equilibrium (Radner (1979), Allen (1981)). Indeed, such an equilibrium yields ex-post Walrasian allocations. Our convergence result for the core suggests that the full revelation of information that accompanies the price function is a consequence of the communication mechanisms - unrestricted random blocking plans- used by coalitions in large economies: an allocation without the market equilibrium 
property does not survive these blocking moves, after such communication devices are employed. That is, a Bayesian equilibrium blocking of some such communication mechanism, in which information is credibly transmitted, knocks that allocation out of the core. The allocations that remain in the core of every replica are therefore immune to any conceivable credible communication, and those happen to be the ex-post Walrasian allocations. On the other hand, the core does not shrink enough if either information transmission is prevented to circulate across different coalitions that participate in a given random blocking plan (e.g., when replicas are independent), or if it is not an equilibrium phenomenon (e.g., when one works with ex-post replicas).

By taking an approach based on the core, the current paper reaches conclusions about informational flows in large economies that are independent of the specific trading procedure. Our findings therefore complement other results in the literature, directly grounded on non-cooperative game-theoretic models, in terms of providing foundations/criticisms of rational expectations equilibria in studies of specific procedures. These include Holden and Subrahmanyan (1992) and Foster and Viswanathan (1996) in models á la Kyle (1985); Dubey, Geanakoplos and Shubik (1987) and Forges and Minelli (1997) in market games á la Shapley and Shubik (1977); Milgrom (1981), Pesendorfer and Swinkels (1997), Reny and Perry (2006) and Peters and Severinov (2008) in auctions; and Wolinsky (1990), Blouin and Serrano (2001) and Gottardi and Serrano (2005) in matching\& bargaining and related models. The latter paper also includes a detailed description of the relevant issues in these and other papers.

\section{Preliminaries}

\subsection{An Interim Exchange Economy}

The basic model of an exchange economy with asymmetric information that we shall use in this paper can be formulated as follows. Let $T_{i}$ denote the (finite) set of agent $i$ 's types. The interpretation is that $t_{i} \in T_{i}$ denotes the private information possessed by agent $i$. With $N=\{1, \ldots, n\}$ as the finite set of agents, let $T_{N}=\prod_{i \in N} T_{i}$ denote the set of all information states. We will use the notation $t_{-i}$ to denote $\left(t_{j}\right)_{j \neq i}$. Similarly $T_{-i}=\prod_{j \neq i} T_{j}$, $T_{S}=\prod_{j \in S} T_{j}$ and $T_{-S}=\prod_{j \notin S} T_{j}$. We assume that agents have a common 
prior probability distribution $q$ defined on $T_{N}$, and that no type is redundant, i.e., $q\left(t_{i}\right)>0$ for all $t_{i} \in T_{i}$ for all $i$. At the interim stage, nature chooses $t_{N} \in T_{N}$, and each agent $i$ knows her type, $t_{i}$. Hence, conditional probabilities will be important: for each $i \in N$ and $t_{i} \in T_{i}$, the conditional probability of $t_{-i} \in T_{-i}$, given $t_{i}$ is denoted $q\left(t_{-i} \mid t_{i}\right)$.

We assume that there are $|L|=l$, a finite number of commodities, and that commodity $L$ is a numeraire. The consumption set of agent $i$ is $X_{i}=$ $R_{+}^{l-1} \times R$. Agent $i$ 's utility function in state $t_{N}$ is denoted $u_{i}\left(\cdot, t_{N}\right): X_{i} \times T_{N} \mapsto$ $R$, and it is quasilinear in the numeraire: $u_{i}\left(x_{i}, t_{N}\right)=v_{i}\left(x_{i}^{-l}, t_{N}\right)+x_{i}^{l}$. The endowment of agent $i$ of type $t_{i}$ is $\omega_{i} \in X_{i}$ (assumed to be independent of the state - with this assumption, all private information concerns agents' preferences and beliefs.)

We can now define an admissible exchange economy as $\mathcal{E}=\left\langle\left(u_{i}, X_{i}, \omega_{i}\right.\right.$, $\left.\left.T_{i}\right)_{i \in N}, q\right\rangle$.

In our analysis, the contracts will be signed at the interim stage. However, when a coalition gets together and considers to upset a given allocation, some information may be transmitted within the members of the coalition. We shall allow coalitions to use random incentive compatible mechanisms, as we explain next.

\subsection{Allocation Rules as Mechanisms}

\subsubsection{Deterministic Mechanisms}

For coalition $S \subseteq N$, a feasible deterministic (state contingent) $S$-allocation, $x: T_{N} \mapsto R^{l s}$ (where $s$ denotes the cardinality of $S$ ), consists of a commodity bundle for each consumer in $S$ in each state such that $\sum_{i \in S} x_{i}\left(t_{N}\right) \leq \sum_{i \in S} \omega_{i}$ for all $t_{N} \in T_{N}$, and satisfying that $x\left(t_{S}, t_{-S}^{\prime}\right)=x\left(t_{S}, t_{-S}^{\prime \prime}\right)$ for all $t_{S} \in T_{S}$ and for all $t_{-S}^{\prime}, t_{-S}^{\prime \prime} \in T_{-S}$. (The latter assumption is made to exclude basic externalities across coalitions, i.e., the set of feasible allocations to a coalition is independent of the information held by the complement, although this may affect the utilities of agents in the coalition). We will denote by $\mathcal{A}_{S}$ the set of feasible deterministic state contingent allocations of $S$. With confusion being avoided by the context, we shall also use $\mathcal{A}_{S}$ to denote the set of feasible deterministic allocations in a given state: $\mathcal{A}_{S}=\left\{\left(x_{i}\right) \in \prod_{i \in S} X_{i} \mid \sum_{i \in S} x_{i} \leq\right.$ $\left.\sum_{i \in S} \omega_{i}\right\}$. Similarly, deterministic state contingent $N$-allocations are simply referred to as deterministic allocations, and the set of such deterministic allocations is denoted by $\mathcal{A}_{N}$. 
For much of this paper, we shall work in environments in which the information state will not be verifiable, not even at the ex-post stage. Thus, it becomes necessary to impose the incentive compatibility constraints into the analysis.

We begin again by considering deterministic allocations. Then, if agent $i$ of type $t_{i}$ pretends to be of type $t_{i}^{\prime}$ (while all other agents are truthful), she gets interim utility:

$$
U_{i}\left(x, t_{i}^{\prime} \mid t_{i}\right)=\sum_{t_{-i} \in T_{-i}} q\left(t_{-i} \mid t_{i}\right) u_{i}\left(x_{i}\left(t_{-i}, t_{i}^{\prime}\right),\left(t_{-i}, t_{i}\right)\right) .
$$

An allocation $x$ is incentive compatible (IC) if for every $i \in N$, and for every $t_{i} \in T_{i}$

$$
U_{i}\left(x \mid t_{i}\right) \geq U_{i}\left(x, t_{i}^{\prime} \mid t_{i}\right)
$$

for every $t_{i}^{\prime} \in T_{i} \backslash\left\{t_{i}\right\}$. We denote the set of IC allocations by $\mathcal{A}_{N}^{*}$. An allocation $x$ is strictly $I C$ if all these inequalities are strict.

Information transmission concerns ruling out some states as impossible, through the identification of smaller events. For an event $E \subseteq T_{N}$ and $t_{i} \in T_{i}$, let

$$
E_{-i}\left(t_{i}\right)=\left\{t_{-i} \in T_{-i} \mid\left(t_{i}, t_{-i}\right) \in E\right\}
$$

and

$$
E_{i}=\left\{t_{i} \in T_{i} \mid E_{-i}\left(t_{i}\right) \neq \emptyset\right\} .
$$

Consider an allocation rule $x \in \mathcal{A}_{N}$, agent $i$ of type $t_{i}$ and an event $E$. Suppose $q\left(E_{-i}\left(t_{i}\right)\right)>0$. Then the interim utility conditional on $E$ can be expressed as:

$$
U_{i}\left(x \mid t_{i}, E\right)=\sum_{t_{-i} \in E_{-i}\left(t_{i}\right)} \frac{q\left(t_{-i} \mid t_{i}\right)}{q\left(E_{-i}\left(t_{i}\right) \mid t_{i}\right)} u_{i}\left(x_{i}\left(t_{-i}, t_{i}\right),\left(t_{-i}, t_{i}\right)\right) .
$$

The corresponding interim utility (conditional on $E$ ) if type $t_{i}$ pretends to be of type $t_{i}^{\prime}$, while the others are truth-telling, is:

$$
U_{i}\left(x, t_{i}^{\prime} \mid t_{i}, E\right)=\sum_{t_{-i} \in E_{-i}\left(t_{i}\right)} \frac{q\left(t_{-i} \mid t_{i}\right)}{q\left(E_{-i}\left(t_{i}\right) \mid t_{i}\right)} u_{i}\left(x_{i}\left(t_{-i}, t_{i}^{\prime}\right),\left(t_{-i}, t_{i}\right)\right) .
$$

Given $E \subseteq T_{N}$, an $S$-allocation $x \in \mathcal{A}_{S}$ is $I C$ over $E$ if for every $i \in S$ and for every $t_{i}, t_{i}^{\prime} \in E_{i}$ :

$$
U_{i}\left(x \mid t_{i}, E\right) \geq U_{i}\left(x, t_{i}^{\prime} \mid t_{i}, E\right)
$$




\subsubsection{Random Mechanisms}

At this point it will be convenient to introduce the notion of a status quo. By a status quo we refer to an IC, state contingent allocation $x \in \mathcal{A}_{N}^{*}$ with the interpretation that in every state $t_{N}$, the outcome is $x\left(t_{N}\right)$, unless there is an agreement to change it. This means, in particular, that if there is an attempt to change the status quo but the attempt fails, the outcome in state $t_{N}$ is $x\left(t_{N}\right)$, i.e., any discussion about a possible change does not by itself allow any agent to strategically manipulate the status quo $x$.

To potentially destabilize a status quo, now we expand coalitional interactions and information transmission to also consider random plans. A random coalitional plan $\mu$ consists of a collection of probability distributions over feasible allocation rules for various coalitions. In particular, $\mu\left(S, y^{S}, t_{N}\right)$, where $y^{S} \in \mathcal{A}_{S}$, denotes the probability with which coalition $S \subseteq N$ is receiving $y^{S} \in \mathcal{A}_{S}$ when the (reported) state is $t_{N} \in T_{N}$.

We shall say that such a random plan is measurable with respect to coalitional information, or simply measurable, if for every $S$, for every $y^{S}$ and for every $t_{S}, \mu\left(S, y^{S},\left(t_{S}, t_{-S}^{\prime}\right)\right)=\mu\left(S, y^{S},\left(t_{S}, t_{-S}^{\prime \prime}\right)\right)$ for every $t_{-S}^{\prime}, t_{-S}^{\prime \prime} \in T_{-S}$. Otherwise, we shall say that the random plan is non-measurable. Nonmeasurabilities in this sense are in principle possible, although we shall always require that, if $P$ is the union of all coalitions in the support of $\mu$, $\mu\left(S, y^{S},\left(t_{P}, t_{-P}^{\prime}\right)\right)=\mu\left(S, y^{S},\left(t_{P}, t_{-P}^{\prime \prime}\right)\right)$ for all $\left(S, y^{S}\right)$ in the support of $\mu$, all $t_{P} \in T_{P}$ and all $t_{-P}^{\prime}, t_{-P}^{\prime \prime} \in T_{-P}$. The idea is to think of the random plan as a mediated communication mechanism used by the members of the coalition; thus, information can potentially flow in any possible way within the mechanism, but only information available to someone that participates in the random plan.

Note that, if $\mu\left(S, y^{S}, t_{N}\right)>0$ implies $\mu\left(S, z^{S}, t_{N}\right)=0$ for all $z^{S} \neq y^{S}$, we would associate with each coalition $S$ one deterministic allocation $y^{S} \in \mathcal{A}_{S}$. Also, if $\mu\left(S^{\prime}, \cdot, \cdot\right)=0$ for every $S^{\prime} \neq S$, the plan relies only on a single coalition. But in general a random plan may include random allocations within each coalition, as well as random coalition formation. The plan specifies for each $t_{N} \in T_{N}, 0 \leq \mu\left(S, y^{S}, t_{N}\right) \leq 1$ for all coalitions $S$ and $S$-allocations $y^{S}$, and $\sum_{S} \sum_{y^{S}} \mu\left(S, y^{S}, t_{N}\right) \leq 1$.

For type $t_{i}$ of agent $i$, the interim utility of such a random plan $\mu$, completed with the implementation of the status quo $x$ as needed when the 
probabilities of the plan do not add up to 1, is this:

$$
\begin{aligned}
U_{i}\left([\mu / x] \mid t_{i}\right)=\sum_{t_{-i}} q\left(t_{-i} \mid t_{i}\right)\left[\sum_{S \supseteq\{i\}} \sum_{y^{S}} \mu\left(S, y^{S}, t_{N}\right) u_{i}\left(y_{i}^{S}\left(t_{N}\right), t_{N}\right)\right. & \\
& \left.+\nu_{i}\left(t_{N}\right) u_{i}\left(x_{i}\left(t_{N}\right), t_{N}\right)\right],
\end{aligned}
$$

where $\nu_{i}\left(t_{N}\right):=1-\sum_{S \supseteq\{i\}} \sum_{y^{S}} \mu\left(S, y^{S}, t_{N}\right) .^{5}$

If everyone else is truthful and type $t_{i}$ pretends to be type $t_{i}^{\prime}$, his interim expected utility from the random plan $\mu$ completed with the status quo $x$ is:

$$
\begin{array}{r}
U_{i}\left([\mu / x], t_{i}^{\prime} \mid t_{i}\right)=\sum_{t_{-i}} q\left(t_{-i} \mid t_{i}\right)\left[\sum_{S \supseteq\{i\}} \sum_{y^{S}} \mu\left(S, y^{S},\left(t_{-i}, t_{i}^{\prime}\right)\right) u_{i}\left(y_{i}^{S}\left(t_{-i}, t_{i}^{\prime}\right), t_{N}\right)\right. \\
\left.+\nu_{i}\left(t_{-i}, t_{i}^{\prime}\right) u_{i}\left(x_{i}\left(t_{N}\right), t_{N}\right)\right]
\end{array}
$$

where $\nu_{i}\left(t_{i}^{\prime}, t_{-i}\right):=1-\sum_{S \supseteq\{i\}} \sum_{y^{S}} \mu\left(S, y^{S},\left(t_{i}^{\prime}, t_{-i}\right)\right)$.

Note how the type misreport affects the implementation probabilities $\mu(\cdot)$ of each coalitional allocation, and therefore also $\nu_{i}(\cdot)$ of the status quo. In addition, it garbles the outcomes in each $y^{S}$, but not those in $x$. In the next subsection, we shall go over this, which is related to the timing of events in the blocking plan versus the status quo; see also Myerson (2007) and Serrano and Vohra (2007).

A random plan $\mu$ is $I C$ if for every $i \in N$ and for every $t_{i}, t_{i}^{\prime}$ in the support of $\mu$ :

$$
U_{i}\left([\mu / x] \mid t_{i}\right) \geq U_{i}\left([\mu / x], t_{i}^{\prime} \mid t_{i}\right)
$$

\subsection{Core w.r.t. Equilibrium Blocking}

The crux of this paper is the endogenous information transmission that goes on within a coalition of asymetrically informed agents (unlike, say, the concepts of coarse and fine core in Wilson's (1978) seminal work). To study such endogenous information transmission, the concepts presented so far can be used to define appropriate versions of the core. It is important to remark that, when coupled with an IC allocation $x \in \mathcal{A}_{N}^{*}$, interpreted as the status quo, a random plan trying to upset it may allow for the status quo to prevail with some positive probability. That is, since a random plan $\mu$ against the

\footnotetext{
${ }^{5}$ More precisely, if a random plan $\mu$ includes random allocations within each coalition, then $\mu$ might have uncountable support, and hence, the interim utility should be defined incorporating this. For simplicity, we assume that $\mu$ allows only countable support. None of our arguments rely on this assumption.
} 
presence of status quo $x$ allows for each $t_{N} \in T_{N}, 0 \leq \mu\left(S, y^{S}, t_{N}\right) \leq 1$ for all coalitions $S$ and $S$-allocations $y^{S}$, and $\sum_{S} \sum_{y^{S}} \mu\left(S, y^{S}, t_{N}\right) \leq 1$, one must assign the rest of probability $\nu\left(t_{N}\right)$ to the implementation of the status quo. ${ }^{6}$

We next define a random plan as a communication mechanism. Following Myerson (2007) and Serrano and Vohra (2007), the discussions of a blocking plan against a status quo in a context of incomplete information take place after the truthful reports for the status quo have occurred, but before the implementation of such a status quo. Therefore, one can specify the following timing of actions within the blocking plan $\mu$ against an IC $x$ :

- Stage 0: types are reported to sustain $x$ as an IC allocation. These type reports $t_{N}^{0}$ will be used every time the status quo needs to be implemented, and their reports are made with independence of any potential blocking plan. ${ }^{7}$ This stage is therefore not part of the random plan as a communication mechanism.

- Stage 1: this and the next stages describe the timing of actions within the blocking plan $\mu$ itself. Types are reported again after players have been informed about $\mu$. Each player is privately informed about the instances in which $\mu$ calls him to act. The type reports of this stage are used only if and when the blocking plan is implemented.

- Stage 2: players are invited to participate in the blocking plan, i.e., phone calls are made by the blocking mediator, taking into account the probabilities $\mu\left(S, y^{S}, t_{N}\right)$, which use already the types reported within $\mu$. Note in particular how a non-measurable plan is perfectly possible, allowing information transmission from coalition to coalition within $\mu$. On the other hand, the plan must be measurable with respect to the information of the union of coalitions within $\mu$.

- Stage 3: each agent in the support of $\mu$ is asked to either accept or reject the blocking plan, and he does so in private communications with the blocking mediator. An allocation proposed to coalition $S$ is

\footnotetext{
${ }^{6}$ When the appropriate payoff inequalities are imposed versus a given status quo, one describes a "blocking plan" used in the definition and characterization of the inner core; see Myerson (1991), Qin (1993) and de Clippel and Minelli (2005).

${ }^{7}$ If this is not the case and there is complete forward-looking behavior, i.e., agents envisioning each possible blocking plan before they report their types to sustain the status quo, one would probably arrive at a concept far afield from the core.
} 
implemented if and only if every agent in $S$ accepts the random plan; otherwise, the status quo $x\left(t_{N}^{0}\right)$ is implemented.

Two observations are in order that justify this communication mechanism, in terms of how it would work under complete information. First, in a complete information economy, the arrangements behind the status quo and those behind a blocking move are kept "separate," in the sense that agents believe that if the blocking move fails, they can get back to the status quo, which is still available as it was before the blocking conversations began. This justifies the use of the reports $t_{N}^{0}$ every time the blocking plan is not approved. Second, a player, being informed only of the probabilities $\mu(\cdot)$ of coalitions that include him, assigns the rest of probability to the status quo prevaling. Again, under complete information, if a player is not called to participate in a blocking move, he believes the status quo will happen (even though it may not be even feasible, once the blocking plan succeeds).

The approach just described is quite general. As explained in Serrano and Vohra (2007), one could conceivably consider arbitrary communication mechanisms, but there is a version of the revelation principle at work. Any equilibrium of such general communication mechanism can be made outcome equivalent to a Bayesian equilibrium of the mechanism just described, in which types are reported truthfully.

We shall say that there is an equilibrium rejection of $x$ if there exists a Bayesian equilibrium of the above communication mechanism in which the plan $\mu$ is accepted with positive probability. ${ }^{8}$ Without loss of generality, an equilibrium rejection of $x$ will be termed a random blocking plan against $x$.

Given an IC allocation $x \in \mathcal{A}_{N}^{*}$, a random plan $\mu$ is a random blocking plan against $x$ whenever for every type $t_{i} \in T_{i}$ :

$$
U_{i}\left([\mu / x] \mid t_{i}\right) \geq U_{i}\left(x \mid t_{i}\right)
$$

with at least one strict inequality, and

$$
U_{i}\left([\mu / x] \mid t_{i}\right) \geq U_{i}\left([\mu / x], t_{i}^{\prime} \mid t_{i}\right)
$$

for all $t_{i}^{\prime} \in T_{i}$.

\footnotetext{
${ }^{8}$ We shall think of such an equilibrium to be in pure strategies. A mixed equilibrium adds another layer of randomization (on top of the uncertainty about the states, the random coalition formation and the random allocations of physical goods). Mixed equilibria can be handled in the approach, but then the expressions written below need an adjustment in the probabilities.
} 
Equivalently, one can normalize probabilities and simplify these inequalities because of identical terms on both sides, and write:

$$
\sum_{t_{-i}} q\left(t_{N}\right) \sum_{S \supseteq\{i\}} \sum_{y^{S}} \mu\left(S, y^{S}, t_{N}\right)\left[u_{i}\left(y_{i}^{S}\left(t_{N}\right), t_{N}\right)-u_{i}\left(x_{i}\left(t_{N}\right), t_{N}\right)\right] \geq 0
$$

for all $t_{i} \in T_{i}$, with at least one strict inequality, and

$$
\begin{gathered}
\sum_{t_{-i}} q\left(t_{N}\right) \sum_{S \supseteq\{i\}} \sum_{y^{S}} \mu\left(S, y^{S}, t_{N}\right)\left[u_{i}\left(y_{i}^{S}\left(t_{N}\right), t_{N}\right)-u_{i}\left(x_{i}\left(t_{N}\right), t_{N}\right)\right] \\
\geq \sum_{t_{-i}} q\left(t_{N}\right) \sum_{S \supseteq\{i\}} \sum_{y^{S}} \mu\left(S, y^{S},\left(t_{-i}, t_{i}^{\prime}\right)\right)\left[u_{i}\left(y_{i}^{S}\left(t_{-i}, t_{i}^{\prime}\right), t_{N}\right)-u_{i}\left(x_{i}\left(t_{N}\right), t_{N}\right)\right] \\
\text { for all } i \in N \text { and } t_{i}, t_{i}^{\prime} \in T_{i} .
\end{gathered}
$$

Thus, information transmission is endogenous in a random blocking plan. The equilibrium rejection of $x$ implies that those types that are part of the objection best-respond to the others by accepting it, whereas those types that are not do not have an incentive to turn their zero probability of participation into a positive one by pretending to be one of the invited types. This is the content of equations $(*)$ and $(* *)$, which respectively express the equilibrium acceptance condition and the IC constraints over the event described by the equilibrium probabilities.

An IC allocation $x \in \mathcal{A}_{N}^{*}$ is in the core w.r.t. equilibrium blocking of some class of random communication mechanisms whenever there does not exist an equilibrium rejection of $x$, i.e., a random blocking plan against $x$, for a communication mechanism in that class.

Particular cases of this definition are the following. We shall progressively enlarge the class of blocking communication mechanisms considered:

An IC allocation $x \in \mathcal{A}_{N}^{*}$ is in the credible core (Dutta and Vohra (2005)) if and only if it is in the core of single-coalition deterministic mechanisms. That is, whenever there does not exist a random blocking plan $\mu$ against $x$ such that $\mu\left(S, y^{S}, t_{N}\right)>0$ only for one coalition $S$ and one deterministic rule $y^{S} \in \mathcal{A}_{S}$, and for $t_{N} \in \prod_{i \in S} E_{i} \times T_{-S}$, where $E_{i}$ is the set of types of agent $i$ in the support of $\mu$. Note how, in particular, the definition of IC over an event $E$ for an $S$-allocation for a fixed coalition $S$, as defined above, applies to these blocking plans.

Thus, in a credible objection to an allocation, a fixed coalition $S$ identifies an information event over which the objection takes place. All the types consistent with that event prefer the objection to the status quo allocation 
after Bayesian updating of their beliefs given the event. Furthermore, no type within the relevant event wants to misrepresent its information. Finally, the types not consistent with the event do not wish to participate in the objection by pretending they are one of the types in it: to see this, in the second condition of a random blocking plan, suppose $t_{i}$ is one of these excluded types and $t_{i}^{\prime}$ one of the types in the support of $\mu$. The latter is what is called the credibility restriction in this objection. Hence the name credible objection. As can be easily seen, all these conditions are particular cases of equations $(*)$ and $(* *)$ above.

An IC allocation $x \in \mathcal{A}_{N}^{*}$ is in the virtual utility core (Myerson (2007)) if and only if it is in the core of measurable random mechanisms. That is, whenever there does not exist a random blocking plan $\mu$ against $x$ that is measurable.

Thus, in these objections, an agent is invited to the blocking plan, but there is a process of random coalition formation. Each agent in the support of $\mu$ gets a phone call inviting him to participate in the blocking plan, and those "phone calls" are made according to $\mu$. On the basis of $\mu$, contingent on the "phone call," each agent updates his interim beliefs using Bayes' rule. Again, given this, each type is part of the equilibrium rejection of $x$ if and only if he wishes to go along with it instead of remaining at the status quo, and the appropriate IC constraints are also imposed given $\mu$ - equations $(*)$ and $\left.{ }^{* *}\right)$. The measurability of the plan implies that a given coalition $S$ cannot be called with different probabilities in two states $\left(t_{S}, t_{-S}^{\prime}\right)$ and $\left(t_{S}, t_{-S}^{\prime \prime}\right)$ that the coalition cannot discern (observe in particular that the Dutta-Vohra credible objections are measurable).

An IC allocation $x \in \mathcal{A}_{N}^{*}$ is in the randomized mediated core (Serrano and Vohra (2007)) if and only if it is in the core of unrestricted random mechanisms. That is, whenever there does not exist a random blocking plan $\mu$ against $x$.

For these objections, the same story applies, except that the non-measurability of $\mu$ allows for information transmission across coalitions within the blocking plan.

Under the assumptions made so far, and if one allows average feasibility in the numeraire (while exact feasibility is required for the other commodities), Myerson (2007) establishes the non-emptiness of the virtual utility core. Furthermore, his existence argument does not use at all the measurability of blocking plans. Therefore, it follows that, under exactly the same assumptions, the core of unrestricted mechanisms is also non-empty. An alternative 
approach to non-emptiness is provided in Dutta and Vohra (2005). In quasilinear economies, assuming that there exists an IC ex-post core allocation, they show that the credible core is non-empty. It is easy to complete their argument to show that the core of unrestricted mechanisms is also non-empty (see the proof of our Proposition 1). That is, adding randomness to blocking plans does not jeopardize the non-emptiness of the core in this case.

The following is an obvious, but useful observation:

Observation 1: The core of unrestricted mechanisms is a subset of the same concept based on measurable mechanisms, itself a subset of the concept if one only uses single-coalition deterministic mechanisms. ${ }^{9}$

When incentive constraints can be dropped, one can still define the corresponding core w.r.t. equilibrium blocking. Then, a core allocation $x \in \mathcal{A}_{N}$, and the equilibrium rejection of $x$ amounts only to condition $\left(^{*}\right)$. As above, as a function of the class of communication mechanisms considered, one can create similar nested sets of allocations. ${ }^{10}$ In this case, it can be shown (Dutta and Vohra (2005), Serrano and Vohra (2007)) that all fine objections can be made credible, and hence, the core of single-coalition deterministic mechanisms coincides with the fine core. Without incentive constraints, nonemptiness results are easier to obtain: in particular, it follows from Dutta and Vohra (2005) that in quasilinear economies this core is non-empty.

We shall use in some of our proofs the following additional observation:

Observation 2: An IC allocation that is in the fine core (which is in the IC fine core) is in the core of single-coalition deterministic mechanisms.

\subsection{Market Equilibrium}

The competitive market equilibrium concept that we shall employ in this paper is the ex-post Walrasian equilibrium: an allocation rule $x$ is an ex-

\footnotetext{
${ }^{9}$ It is easy to relate these notions to Wilson's (see Dutta and Vohra (2005)). If in a credible objection one drops the credibility restriction (the self-selection of types into the objection), one constructs an (IC) fine objection. The IC fine core is the set of IC allocations $x \in \mathcal{A}_{N}^{*}$ immune to IC fine objections. On the other hand, an IC objection based on a common knowledge event for a coalition is an IC coarse objection, which is always credible. The $I C$ coarse core is the set of incentive compatible $x \in \mathcal{A}_{N}^{*}$ against which there is no IC coarse objection. It follows that the IC fine core is a subset of the credible core, itself a subset of the IC coarse core.

${ }^{10}$ If one drops the IC constraints from both the status quo allocations and the objections in the IC coarse core and IC fine core, one arrives at the coarse and fine core definitions (Wilson (1978)).
} 
post Walrasian equilibrium allocation rule if $x\left(t_{N}\right)$ constitutes a Walrasian equilibrium allocation in each $t_{N} \in T_{N}$. We shall assume that there exists an ex-post Walrasian equilibrium allocation rule that is IC. Under this assumption, our negative convergence result extends also to any price-taking equilibrium concept that satisfies Property P, as first suggested in Serrano, Vohra and Volij (2001):

A price-taking equilibrium concept is said to obey Property $P$ if, whenever it is non-empty, in an economy that includes some fully informed agents, each of them receives in equilibrium a bundle that maximizes his ex-post utility over his ex-post budget constraint.

\section{Equilibrium Inclusion in the Core}

In this section we begin by showing that, in our domain of quasilinear economies, the set of ex-post Walrasian allocation rules that satisfy IC is in the core of unrestricted mechanisms. The section ends by extending the result when incentive constraints are dropped.

Proposition 1 Consider a quasilinear exchange economy $\mathcal{E}$ in our admissible class. Let $x^{*} \in \mathcal{A}_{N}^{*}$ be an ex-post Walrasian equilibrium allocation rule satisfying IC. Then, $x^{*}$ is in the core of unrestricted mechanisms.

Proof: By hypothesis, $x^{*}$ is IC. So we need to show that there does not exist any random blocking plan that improves upon $x^{*}$.

Since an ex-post Walrasian allocation is an element of the ex-post core, we can say that for all $S \subseteq N$, all $t_{N} \in T_{N}$ and $y^{S} \in \mathcal{A}_{S}$,

$$
\sum_{i \in S} u_{i}\left(y_{i}^{S}\left(t_{N}\right), t_{N}\right) \leq \sum_{i \in S} u_{i}\left(x_{i}^{*}\left(t_{N}\right), t_{N}\right)
$$

This corresponds to an ex-post objection. Now, consider a random plan $\mu$ (without measurability). This inequality implies that for all $S \subseteq N$, all $y^{S} \in \mathcal{A}_{S}$ and all $t_{N} \in T_{N}$,

$$
\sum_{i \in S} \mu\left(S, y^{S}, t_{N}\right) u_{i}\left(y_{i}^{S}\left(t_{N}\right), t_{N}\right) \leq \sum_{i \in S} \mu\left(S, y^{S}, t_{N}\right) u_{i}\left(x_{i}^{*}\left(t_{N}\right), t_{N}\right) .
$$


Thus,

$$
\begin{aligned}
& \sum_{S \subseteq N} \sum_{y^{S} \in \mathcal{A}_{S}} \sum_{t_{N} \in T_{N}} q\left(t_{N}\right) \sum_{i \in S} \mu\left(S, y^{S}, t_{N}\right) u_{i}\left(y_{i}^{S}\left(t_{N}\right), t_{N}\right) \\
& \quad \leq \sum_{S \subseteq N} \sum_{y^{S} \in \mathcal{A}_{\mathcal{S}}} \sum_{t_{N} \in T_{N}} q\left(t_{N}\right) \sum_{i \in S} \mu\left(S, y^{S}, t_{N}\right) u_{i}\left(x_{i}^{*}\left(t_{N}\right), t_{N}\right) .
\end{aligned}
$$

If a blocking plan $\mu$ existed, we would obtain (recall equation $\left({ }^{*}\right)$ ):

$$
\begin{aligned}
\sum_{i \in N} & \sum_{t_{N}} q\left(t_{N}\right) \sum_{S \supseteq\{i\}} \sum_{y^{S}} \mu\left(S, y^{S}, t_{N}\right)\left[u_{i}\left(y_{i}^{S}\left(t_{N}\right), t_{N}\right)-u_{i}\left(x_{i}^{*}\left(t_{N}\right), t_{N}\right)\right] \\
& =\sum_{S \subseteq N} \sum_{y^{S}} \sum_{t_{N}} q\left(t_{N}\right) \sum_{i \in S} \mu\left(S, y^{S}, t_{N}\right)\left[u_{i}\left(y_{i}^{S}\left(t_{N}\right), t_{N}\right)-u_{i}\left(x_{i}^{*}\left(t_{N}\right), t_{N}\right)\right] \\
& >0
\end{aligned}
$$

which contradicts the inequality in the previous paragraph.

Thus, in quasilinear economies, ex-post Walrasian allocation rules that satisfy IC are elements of the smallest core w.r.t. equilibrium blocking. It is immediate to drop the incentive constraints from this result, and with the same proof (note it does not rely on the IC condition $(* *)$ ), demonstrate the following:

Proposition 2 Consider a quasilinear exchange economy $\mathcal{E}$ in our admissible class, but where incentive constraints are dropped. Let $x^{*} \in \mathcal{A}_{N}$ be an ex-post Walrasian equilibrium allocation rule. Then, $x^{*}$ is in the core of unrestricted mechanisms.

Remark: Ignoring incentive constraints, Dutta and Vohra (2005) show that in quasilinear economies the ex-post core is included in the fine core, which is therefore non-empty. The proof of Proposition 2 strengthens this result, as without incentive constraints, the fine core coincides with the core of singlecoalition deterministic mechanisms.

Remark: Propositions 1 and 2 apply to all quasilinear economies, including their different kinds of replicas, as we are about to define in the next sections. Remark: The conclusions of Propositions 1 and 2 do not extend to nonquasilinear economies. In fact, ex-post Walrasian rules may be even interim inefficient. 


\section{Independent Replica Economies}

We turn to define the replicas of the basic economies and allocations. We shall first use the independent replicas introduced in Gul and Postlewaite (1992), also used in Forges, Heifetz and Minelli (2001). Each agent's utility depends only on the information contained in his replica. Because replicas are independent, the set of states in the replicated economy is the product of the sets of states for each replica.

Formally, given an economy $\mathcal{E}=\left\langle\left(u_{i}, X_{i}, \omega_{i}, T_{i}\right)_{i \in N}, q\right\rangle$, and an allocation $x \in \mathcal{A}_{N}$, independent replicas of $\mathcal{E}$ and $x$ are defined as follows. For every positive integer $m$, let $I_{m}=\{1,2, \ldots, m\}$. The $m$-th replica of $\mathcal{E}$ is the economy $\mathcal{E}^{m}=\left\langle\left(u_{(i, j)}, X_{(i, j)}, \omega_{(i, j)}, T_{(i, j)}\right)_{(i, j) \in N \times I_{m}}, q_{m}\right\rangle$, where for all $(i, j) \in$ $N \times I_{m}, X_{(i, j)}=X_{i}, T_{(i, j)}=T_{i}$ over $\prod_{k \neq i} T_{(k, j)}, \omega_{(i, j)}=\omega_{i}, u_{(i, j)}=u_{i}:$ $X_{i} \times \prod_{i \in N} T_{(i, j)} \mapsto R$, and

$$
q_{m}\left(t_{(1,1)}, \ldots, t_{(n, 1)}, \ldots, t_{(1, m)}, \ldots, t_{(n, m)}\right)=\prod_{j \in I_{m}} q\left(t_{(1, j)}, \ldots, t_{(n, j)}\right) .
$$

The $m$-th replica of $x$ is denoted $x^{m}$ where $x_{(i, j)}^{m}=x_{i}$ for all $(i, j) \in N \times I_{m}$.

We shall say that a set of allocation rules satisfies the equal treatment property if, in any replicated economy, it contains only replicas of allocations $x$ of the basic economy.

Note that the set of information states changes with replication. Since types are independent, each agent potentially retains a piece of private information even after replication, and incentive constraints do not disappear. On the other hand, since preferences depend only on information held by agents in the same copy of the economy, an agent's private information affects a vanishingly small fraction of agents if one replicates the economy sufficiently many times.

\subsection{Non-Convergence Results}

In the current subsection we show that, even if one restricts attention to equal treatment allocations, the core of measurable mechanisms does not converge to any price-taking equilibrium allocations satisfying Property $\mathrm{P}$, no matter how many times the economy is replicated. Later in this subsection we shall argue that this core, in any replicated economy, may in addition contain allocations that violate equal treatment. Thus, if the communication mech- 
anisms used by coalitions are restricted to be measurable random blocking plans, the core remains "too large."

\subsubsection{Equal Treatment Allocations}

Our first non-convergence result follows:

Proposition 3 Consider independent replicas. There exists an allocation $\tilde{x} \in \mathcal{A}_{N}^{*}$ in an admissible economy $\mathcal{E}$ satisfying that for every $m, \tilde{x}^{m}$ is in the core of measurable mechanisms in the replicated economy $\mathcal{E}^{m}$ and that cannot be supported by any price-taking equilibrium notion that obeys Property $P$.

Proof: Consider the following economy $\mathcal{E}$. There are two consumers and two commodities. Suppose $T_{1}=\{s, t\}$ while agent 2 is uninformed (and therefore has only one type). The information state can then be described by $s$ or $t$. Suppose $s$ and $t$ are equally probable. Let $\omega_{1}=\omega_{2}=(1.5,1)$. The utility functions are as follows:

$$
\begin{array}{ll}
u_{1}\left(x^{1}, x^{2}, s\right)=\ln x^{1}+x^{2}, & u_{2}\left(x^{1}, x^{2}, s\right)=2 \ln x^{1}+x^{2} \\
u_{1}\left(x^{1}, x^{2}, t\right)=2 \ln x^{1}+x^{2}, & u_{2}\left(x^{1}, x^{2}, t\right)=\ln x^{1}+x^{2} .
\end{array}
$$

(Recall that throughout we use superscripts to index commodities and subscripts to index consumers.) Thus, the two individuals are ex-ante identical, but the realized type of individual 1 determines ex-post which of the two has a higher utility from consumption of the first good. ${ }^{11}$

We consider first the allocation $\hat{x}$ defined by:

$$
\begin{array}{ll}
\hat{x_{1}}(s)=(1,1.5), & \hat{x_{2}}(s)=(2,0.5) ; \\
\hat{x_{1}}(t)=(2,0.5), & \hat{x_{2}}(t)=(1,1.5) .
\end{array}
$$

Then each agent's ex-post utility is:

$$
\begin{array}{ll}
u_{1}\left(\hat{x_{1}}(s), s\right)=1.5, & u_{2}\left(\hat{x_{2}}(s), s\right)=2 \ln 2+0.5 \\
u_{1}\left(\hat{x_{1}}(t), t\right)=2 \ln 2+0.5, & u_{2}\left(\hat{x_{2}}(t), t\right)=1.5
\end{array}
$$

\footnotetext{
${ }^{11}$ This example was first proposed in Kreps (1977) to illustrate how the set of rational expectations equilibria might be empty. It was also used in Forges, Heifetz and Minelli (2001) to show that the equal treatment property does not hold for the ex-ante incentive compatible core in the two-fold replicated economy.
} 
Note how $\hat{x}$ must be the only allocation prescribed by an equilibrium concept that obeys Property $\mathrm{P}$ in this economy. Note also how many equilibrium concepts will obey this property here, including the ex-post Walrasian equilibrium, the constrained market equilibrium found in Wilson (1978), or the rational expectations equilibrium (which here yields the empty set). Finally, observe that $\hat{x}$ is IC.

To construct the allocation we are interested in, we modify $\hat{x}$ by requiring an additional transfer of 0.05 units of good 2 from individual 2 to individual 1 in each state. We denote the resulting allocation as $\tilde{x}$ :

$$
\begin{array}{ll}
\tilde{x_{1}}(s)=(1,1.55), & \tilde{x_{2}}(s)=(2,0.45) ; \\
\tilde{x_{1}}(t)=(2,0.55), & \tilde{x_{2}}(t)=(1,1.45) .
\end{array}
$$

This allocation yields utility levels

$$
\begin{array}{ll}
u_{1}\left(\tilde{x_{1}}(s), s\right)=1.55, & u_{2}\left(\tilde{x_{2}}(s), s\right)=2 \ln 2+0.45 \\
u_{1}\left(\tilde{x_{1}}(t), t\right)=2 \ln 2+0.55, & u_{2}\left(\tilde{x_{2}}(t), t\right)=1.45
\end{array}
$$

We consider now the independent replication process described above. Recall that we denote individual $i$ in the $j$-th replica as $(i, j)$. We present our argument in two claims.

Claim 3.1: For all $m$, both the $m$-th replicated allocations $\hat{x}^{m}$ and $\tilde{x}^{m}$ are in the core of single-coalition deterministic mechanisms in $\mathcal{E}^{m}$.

Proof of Claim 3.1: We will show that the $m$-th replication of $\tilde{x}$ is in the core of single-coalition deterministic mechanisms in the replicated economy $\mathcal{E}^{m}$. (The argument for $\hat{x}^{m}$ is similar and we omit it.)

Since we can check that $\tilde{x}^{m}$ is IC (each informed agent in each replica does not wish to pretend that he is of the other informed type), it suffices to show that $\tilde{x}^{m}$ is in the fine core of $\mathcal{E}^{m}$; see Observation 2 above.

It is easy to show that $\tilde{x}$ is maximizing the sum of ex-post utilities for each state and it is ex-post individually rational. Hence, $\tilde{x}$ is in the fine core of the original economy $\mathcal{E}$. Thus, for $j=1,2, \ldots, m$, if the allocation is blocked by a coalition $S$ that includes both $(1, j)$ and $(2, j)$, then the allocation is also blocked by $S \backslash\{(1, j),(2, j)\}$. This implies that it is sufficient to check whether coalition $S=\{(1,1), \ldots,(1, k),(2, k+1), \ldots,(2, m)\}$ blocks the allocation $\tilde{x}^{m}$ for each $m$ and $k(0 \leq k \leq m)$. For this possible blocking coalition, we can restrict attention to information events of the form $E=$ $\prod_{j=1}^{k}\left\{r_{(1, j)}\right\} \times \prod_{j=k+1}^{m} T_{(1, j)}$, where $r_{(1, j)}=s$ or $t(1 \leq j \leq k)$. 
Without loss of generality, we may assume that

$$
r_{(1, j)}= \begin{cases}s & \left(1 \leq j \leq j^{\prime}\right) \\ t & \left(j^{\prime}+1 \leq j \leq k\right)\end{cases}
$$

for some $j^{\prime}\left(0 \leq j^{\prime} \leq k\right)$. Here, $j^{\prime}=0\left(j^{\prime}=k\right)$ means that $r_{(1, j)}=t(s)$ for every $(1, j)(1 \leq j \leq k)$, respectively.

Note that any feasible allocation for coalition $S$ has to be constant on $E$, i.e., each uninformed agent must receive the same bundle in both relevant states. It follows from the quasilinearity that the optimal allocation of good 1 can be obtained as the solution to

$$
\begin{gathered}
\max \sum_{j=1}^{j^{\prime}} \ln x_{(1, j)}^{1}+\sum_{j=j^{\prime}+1}^{k} 2 \ln x_{(1, j)}^{1}+\sum_{j=k+1}^{m} \frac{1}{2}\left(2 \ln x_{(2, j)}^{1}+\ln x_{(2, j)}^{1}\right) \\
\text { s.t. } \sum_{j=1}^{k} x_{(1, j)}^{1}+\sum_{j=k+1}^{m} x_{(2, j)}^{1} \leq 1.5 m .
\end{gathered}
$$

By the first-order condition, the solution of this problem is

$$
x_{(1, i)}^{1}=\left\{\begin{array}{ll}
\lambda & \left(1 \leq j \leq j^{\prime}\right) \\
2 \lambda & \left(j^{\prime}+1 \leq j \leq k\right)
\end{array}, x_{(2, j)}^{1}=1.5 \lambda(k+1 \leq j \leq m),\right.
$$

where

$$
\lambda=\frac{1.5 m}{j^{\prime}+2\left(k-j^{\prime}\right)+1.5(m-k)}=\frac{1.5 m}{1.5 m+0.5 k-j^{\prime}} .
$$

Then the sum of interim utilities is

$$
j^{\prime} \cdot \ln \lambda+\left(k-j^{\prime}\right) \cdot 2 \ln (2 \lambda)+(m-k) \cdot 1.5 \ln (1.5 \lambda)+m .
$$

On the other hand, the sum of interim utilities of the original allocation $\tilde{x}^{m}$ for the coalition is

$$
1.55 j^{\prime}+(2 \ln 2+0.55)\left(k-j^{\prime}\right)+\frac{1}{2}\{(2 \ln 2+0.45)+1.45\} \cdot(m-k) .
$$

Now it suffices to show that (2) - (1) is non-negative for all $m, k(0 \leq k \leq$ $m)$ and $j^{\prime}\left(0 \leq j^{\prime} \leq k\right)$. We denote the difference $[(2)-(1)]$ as $g\left(j^{\prime}, k, m\right)$.

For fixed $k$ and $m(0 \leq k \leq m)$, the first-order condition with respect to the variable $j^{\prime}$ is

$$
\ln (1.5 m)-\ln \left(1.5 m+0.5 k-j^{\prime}\right)
$$


Thus the function $g$ is minimized at $j^{\prime}=0.5 k$. Then

$$
\begin{aligned}
g(0.5 k, k, m) & =1.05 k+(\ln 2+0.95)(m-k)-1.5(m-k) \ln 1.5-m \\
& =(\ln 2-1.5 \ln 1.5-0.05)(m-k)+0.05 k \geq 0
\end{aligned}
$$

(since $\ln 2-1.5 \ln 1.5-0.05>0$ and $m \geq k \geq 0$ ). Therefore, $g\left(j^{\prime}, k, m\right) \geq 0$ for every $j^{\prime}, k$ and $m\left(0 \leq k \leq m, 0 \leq j^{\prime} \leq k\right)$. Thus, the proof of Claim 3.1 is complete.

Claim 3.2: For all $m$, both the $m$-th replicated allocations $\hat{x}^{m}$ and $\tilde{x}^{m}$ are in the core of measurable mechanisms in $\mathcal{E}^{m}$.

Proof of Claim 3.2: We will show that the $m$-th replication of $\tilde{x}$ is in the core of measurable mechanisms in the replicated economy $\mathcal{E}^{m}$. (The argument for $\hat{x}^{m}$ is similar and we omit it.)

In the proof of Claim 3.1, we have argued that the $m$-th replication of $\tilde{x}$ is in the fine core. Therefore, we have shown that for all $S \subseteq N \times I_{m}$, all $t_{S} \in T_{S}$ and all $y^{S} \in \mathcal{A}_{S}$,

$$
\sum_{i \in S} \sum_{t_{-S} \in T_{-S}} q_{m}\left(t_{-S}\right) u_{i}\left(y_{i}^{S}\left(t_{N \times I_{m}}\right), t_{N}\right) \leq \sum_{i \in S} \sum_{t_{-S} \in T_{-S}} q_{m}\left(t_{-S}\right) u_{i}\left(\tilde{x}_{i}\left(t_{N \times I_{m}}\right), t_{N}\right) .
$$

(Recall that $q_{m}$ is the probability distribution over the states in the replicated economy $\left.\mathcal{E}^{m}\right)$. The earlier inequality corresponds to a fine objection in which all information within the coalition is transmitted to its members. Now, consider a random plan $\mu$ that satisfies $T^{S}$-measurability. This inequality implies that for all $S \subseteq N \times I_{m}$, all $y^{S} \in \mathcal{A}_{S}$ and all $t_{S} \in T_{S}$,

$$
\begin{aligned}
& \sum_{i \in S} \mu\left(S, y^{S}, t_{N \times I_{m}}\right) \sum_{t_{-S} \in T_{-S}} q_{m}\left(t_{-S}\right) u_{i}\left(y_{i}^{S}\left(t_{N \times I_{m}}\right), t_{N}\right) \\
& \quad \leq \sum_{i \in S} \mu\left(S, y^{S}, t_{N \times I_{m}}\right) \sum_{t_{-S} \in T_{-S}} q_{m}\left(t_{-S}\right) u_{i}\left(\tilde{x}_{i}\left(t_{N \times I_{m}}\right), t_{N}\right) .
\end{aligned}
$$

Thus,

$$
\begin{aligned}
& \sum_{t_{S} \in T_{S}} q_{m}\left(t_{S}\right) \sum_{i \in S} \mu\left(S, y^{S}, t_{N \times I_{m}}\right) \sum_{t_{-S} \in T_{-S}} q_{m}\left(t_{-S}\right) u_{i}\left(y_{i}^{S}\left(t_{N \times I_{m}}\right), t_{N}\right) \\
& \quad \leq \sum_{t_{S} \in T_{S}} q_{m}\left(t_{S}\right) \sum_{i \in S} \mu\left(S, y^{S}, t_{N \times I_{m}}\right) \sum_{t_{-S} \in T_{-S}} q_{m}\left(t_{-S}\right) u_{i}\left(\tilde{x}_{i}\left(t_{N}\right), t_{N}\right),
\end{aligned}
$$


which implies that

$$
\begin{aligned}
& \sum_{S \subseteq N \times I_{m}} \sum_{y^{S} \in \mathcal{A}_{S}} \sum_{t_{N \times I_{m}} \in T_{N \times I_{m}}} q_{m}\left(t_{N \times I_{m}}\right) \sum_{i \in S} \mu\left(S, y^{S}, t_{N \times I_{m}}\right) u_{i}\left(y_{i}^{S}\left(t_{N \times I_{m}}\right), t_{N}\right) \\
& \leq \sum_{S \subseteq N \times I_{m}} \sum_{y^{S} \in \mathcal{A}_{\mathcal{S}}} \sum_{t_{N \times I_{m}} \in T_{N \times I_{m}}} q_{m}\left(t_{N \times I_{m}}\right) \sum_{i \in S} \mu\left(S, y^{S}, t_{N \times I_{m}}\right) u_{i}\left(\tilde{x}_{i}\left(t_{N}\right), t_{N}\right) .
\end{aligned}
$$

If a blocking plan $\mu$ existed, we would obtain (recall equation $\left({ }^{*}\right)$ ):

$$
\begin{aligned}
& \sum_{i \in N \times I_{m}} \sum_{t_{N \times I_{m}}} q_{m}\left(t_{N \times I_{m}}\right) \sum_{S \supseteq\{i\}} \sum_{y^{S}} \mu\left(S, y^{S}, t_{N \times I_{m}}\right)\left[u_{i}\left(y_{i}^{S}\left(t_{N \times I_{m}}\right), t_{N}\right)\right. \\
& \left.=\sum_{S \subseteq N \times I_{m}} \sum_{y^{S}} \sum_{t_{N \times I_{m}}} q_{m}\left(t_{N \times I_{m}}\right) \sum_{i \in S} \mu\left(\tilde{x}_{i}\left(t_{N}\right), t_{N}\right)\right] \\
& >0,
\end{aligned}
$$

which contradicts the inequality in the previous paragraph.

Claims 3.1 and 3.2 complete the proof of Proposition 3.

Remark: Note the parallel steps followed by the proofs of Claim 3.2 and of Proposition 1. There is an important difference, though. While the allocation $\tilde{x}^{m}$ of the proof of Claim 3.2 is in the fine core of every replica, if $x^{*}$ is IC ex-post Walrasian, $x^{* m}$ is in the ex-post core of every replica. This difference matters: in the former case, the non-existence of a fine objection leads, through the use of measurable blocking plans, to the non-existence of a measurable equilibrium blocking. In the case of the latter, not having ex-post objections in any state leads, through the use of (measurable or not) blocking plans, to the non-existence of any kind of equilibrium blocking.

If incentive constraints are not relevant (as in many general equilibrium analyses, and also in Wilson's (1978) original paper), one can state a similar result to Proposition 3, using an identical proof. The observation is simple enough: the proofs of Claims 3.1 and 3.2 do not rely on IC conditions (recall Proposition 2 for the inclusion of the ex-post Walrasian allocations in the core of unrestricted mechanisms):

Proposition 4 Consider independent replicas and suppose incentive constraints are dropped. There exists an allocation $\tilde{x} \in \mathcal{A}_{N}$ in an admissible 
economy $\mathcal{E}$ satisfying that for every $m, \tilde{x}^{m}$ is in the core of measurable mechanisms in the replicated economy $\mathcal{E}^{m}$ and that cannot be supported by any price-taking equilibrium notion that obeys Property P.

Proof: Consider the same economy as in the proof of Proposition 3. Clearly, even if non-IC random blocking plans are possible, no such blocking exists for $\tilde{x}$. Note how the proof of Proposition 3 makes no use of conditions $(* *)$.

\subsubsection{Non-Equal Treatment Allocations}

In this subsection we demonstrate that the core of measurable mechanisms in replicated economies also contains allocations that violate equal treatment across replicas, even if the economy is replicated an arbitrary number of times.

Indeed, in the same economy used in the proof of Proposition 3, consider the allocation $(\hat{x}, \ldots, \hat{x}, \tilde{x})$. We shall show that this allocation belongs to the core of measurable mechanisms in the $(m+1)$-fold replicated economy $\mathcal{E}^{m+1}$ for all $m \geq 1$. That is, in the first $m$ replicas, the bundles in $\hat{x}$ are allocated, whereas those in $\tilde{x}$ are assigned in the last replica, thereby violating the equal treatment property of this core.

We follow analogous steps to those in Claim 3.1, and first show that this allocation is in the core of single-coalition deterministic mechanisms in the $(m+1)$-replicated economy. Since we can easily check that the proposed allocation is IC, it suffices to show that it is in the fine core. ${ }^{12}$

Now we make the following observations:

Observation 3: For each state, both $\hat{x}$ and $\tilde{x}$ are maximizing the sum of ex-post utilities. Both allocations are in the fine core of the original economy. Observation 4: For $k=1,2, \ldots, m, m+1$, if the allocation is blocked by a coalition $S$ that includes both $(1, k)$ and $(2, k)$, then the allocation is also blocked by $S \backslash\{(1, k),(2, k)\}$.

Observation 5: For $k=1,2, \ldots, m$, if the allocation is blocked by a coalition that includes $(2, k)$ but does not include $(1, k)$, then the allocation is also blocked by the coalition that includes $(1, k)$ but does not include $(2, k)$ (by symmetry of allocation $\hat{x}$ ).

\footnotetext{
${ }^{12}$ Again, with the same example, we are showing that this core violates equal treatment in economies in which incentive constraints are dropped.
} 
¿From these observations, we can say that it is sufficient to check whether coalition $S$ is one of two possibilities: indeed, either $S=\{(1,1), \ldots,(1, m)$, $(2, m+1)\}$ or $S=\{(1,1), \ldots,(1, m),(1, m+1)\}$ block the proposed allocation for each $m$.

For the first possible blocking coalition, we can confine our attention to events of the form $E=\prod_{i \in S}\left\{r_{i}\right\} \times T_{-S}$, where $r_{i}=s$ or $t(i \in S \backslash\{(2, m+1)\})$.

Without loss of generality, we may assume that

$$
r_{(1, i)}= \begin{cases}s & (1 \leq i \leq j) \\ t & (j+1 \leq i \leq m)\end{cases}
$$

for some $j(0 \leq j \leq m)$. Here, $j=0(j=m)$ means that the types of all individuals in $S \backslash\{(2, m+1)\}$ are $s(t)$, respectively.

Note that any feasible allocation has to be constant on $E$ and utility of every individual other than $(2, m+1)$ is determined on $E$. It follows from quasilinearity that the optimal allocation of good 1 can be obtained as the solution to

$$
\begin{gathered}
\max \sum_{i=1}^{j} \ln x_{(1, i)}^{1}+\sum_{i=j+1}^{m} 2 \ln x_{(1, i)}^{1}+\frac{1}{2} \ln x_{(2, m+1)}^{1}+\frac{1}{2} \cdot 2 \ln x_{(2, m+1)}^{1} \\
\text { s.t. } \sum_{i=1}^{m} x_{(1, i)}^{1}+x_{(2, m+1)}^{1} \leq 1.5(m+1) .
\end{gathered}
$$

By the first-order condition, the solution of this problem is

$$
x_{(1, i)}^{1}= \begin{cases}\lambda & (1 \leq i \leq j) \\ 2 \lambda & (j+1 \leq i \leq m)\end{cases}
$$

and $x_{(2, m+1)}^{1}=1.5 \lambda$, where

$$
\lambda=\frac{1.5(m+1)}{j+2(m-j)+1.5} .
$$

Then the sum of interim utilities is:

$$
j \cdot \ln \lambda+(m-j) \cdot 2 \ln (2 \lambda)+1.5 \ln (1.5 \lambda)+(m+1) .
$$

On the other hand, the sum of interim utilities of the original allocation is:

$$
1.5 j+(2 \ln 2+0.5)(m-j)+\frac{1}{2}\{(2 \ln 2+0.45)+1.45\} .
$$


Now it suffices to show that (4) - (3) is non-negative for all $m$ and $j(0 \leq$ $j \leq m)$. We write the difference:

$$
\begin{aligned}
& 1.5 j+(2 \ln 2+0.5)(m-j)+\frac{1}{2}\{(2 \ln 2+0.45)+1.45\} \\
& \quad-[j \cdot \ln \lambda+(m-j) \cdot 2 \ln (2 \lambda)+1.5 \ln (1.5 \lambda)+(m+1)]
\end{aligned}
$$

Take partial derivative with respect to $j$ :

$$
\begin{aligned}
1- & 2 \ln 2-\ln \lambda+2 \ln (2 \lambda)-(2 m-j+1.5)[\partial \ln \lambda / \partial j] \\
& =1+\ln \lambda-(2 m-j+1.5)[\partial \ln \lambda / \partial j] \\
& =\ln \lambda=\ln \left[\frac{1.5 m+1.5}{2 m-j+1.5}\right]
\end{aligned}
$$

which vanishes at $j=0.5 \mathrm{~m}$, is negative for $j<0.5 \mathrm{~m}$ and positive thereafter. Therefore, it suffices to check the value of the function at $j=0.5 \mathrm{~m}$ for a fixed $m$ :

$$
\begin{aligned}
0.75 m+(2 \ln 2+0.5)(0.5 m)+ & \frac{1}{2}\{(2 \ln 2+0.45)+1.45\} \\
& -[(0.5 m) \cdot 2 \ln 2+1.5 \ln (1.5)+(m+1)] \\
= & \ln 2 m+\frac{1}{2}\{(2 \ln 2+0.45)+1.45\}-[(0.5 m) \cdot 2 \ln 2+1.5 \ln (1.5)+1] \\
= & \ln 2 m+\ln 2+0.95-[\ln 2 m+1.5 \ln (1.5)+1] \\
= & \ln 2-0.05-[1.5 \ln (1.5)]=0.0340 \ldots>0 .
\end{aligned}
$$

Since this value is independent of $m$, we have shown that for all $j$ satisfying that $0 \leq j \leq m$ for all $m \geq 1$, the difference (4) - (3) is positive, which contradicts that the coalition blocks the original allocation.

If the blocking coalition contains agent $(1, m+1)$ instead of $(2, m+1)$, one can have two cases:

First, it is agent $(1, m+1)$ in state $s$. We write the utility difference, similar to $(4)-(3)$, for this case:

$$
\begin{aligned}
& 1.5 j+(2 \ln 2+0.5)(m-j)+1.55 \\
& \quad-\quad[(j+1) \cdot \ln \lambda+(m-j) \cdot 2 \ln (2 \lambda)+(m+1)]
\end{aligned}
$$

and now $\lambda=(1.5 m+1.5) /(2 m-j+1)$. 
Again, we partially differentiate the aggregate utility difference with respect to $j$ :

$$
\begin{aligned}
1- & 2 \ln 2+\ln \lambda+2 \ln 2-(2 m-j+1)[\partial \ln \lambda / \partial j] \\
= & \ln \lambda=\ln \left[\frac{1.5 m+1.5}{2 m-j+1}\right],
\end{aligned}
$$

which vanishes at $j=0.5 m-0.5$, is negative if $j<0.5 m-0.5$ and positive thereafter.

We thus check the value of the aggregate utility difference at $j=0.5 m-$ 0.5 :

$$
\begin{aligned}
1.5(0.5 m-0.5)+(2 \ln 2+0.5)(0.5 m+0.5)+1.55 & -[(0.5 m+0.5) 2 \ln 2+(m+1)] \\
= & 0.75 m-0.75+\ln 2(m+1)+0.25 m+0.25+1.55-[(m+1)(1+\ln 2)] \\
= & 0.05>0,
\end{aligned}
$$

and also independent of $m$, so we are also done with this case. Finally, we have case 2 : agent $(1, m+1)$ acts in state $t$. The utility difference similar to $(2)-(1)$ is now:

$$
\begin{aligned}
& 1.5 j+(2 \ln 2+0.5)(m-j)+2 \ln 2+0.55 \\
& \quad-\quad[j \cdot \ln \lambda+(m+1-j) \cdot 2 \ln (2 \lambda)+(m+1)],
\end{aligned}
$$

where now $\lambda=(1.5 m+1.5) /(2 m-j+2)$.

Differentiating partially with respect to $j$, one gets:

$$
\begin{aligned}
1 & -2 \ln 2-[\ln \lambda-2 \ln (2 \lambda)+(2 m-j+2)(\partial \ln \lambda / \partial j)] \\
& =-2 \ln 2-[\ln \lambda-2 \ln (2 \lambda)] \\
& =\ln \lambda=\ln \left[\frac{1.5 m+1.5}{2 m-j+2}\right]
\end{aligned}
$$

which vanishes at $j=0.5 m+0.5$, is negative at $j<0.5 m+0.5$, and positive thereafter. Therefore, it suffices to check the value of the aggregate utility difference at $j=0.5 m+0.5$ :

$$
\begin{aligned}
& 1.5(0.5 m+0.5)+(2 \ln 2+0.5)(0.5 m-0.5) \\
& +2 \ln 2+0.55-[(m+1)(\ln 2+1)] \\
& =0.05>0,
\end{aligned}
$$


also independent of $m$.

Thus, now the proof is complete. No such blocking coalition exists and the proposed allocation is in the IC fine core, and hence, also in the core of single-coalition deterministic mechanisms (Observation 2).

The arguments to show that the allocation is in the core of measurable mechanisms are similar to the inequalities derived in Claim 3.2, and we therefore omit them.

\subsection{Convergence of the Core of Unrestricted Mecha- nisms}

In this subsection we show that the allocations of the core of unrestricted mechanisms converge to the set of ex-post Walrasian allocations as the economy is replicated enough times. Thus, in a large enough replica, unrestricted random blocking plans are capable of knocking out any allocation without the ex-post market equilibrium property. Recall that the inclusion of equilibrium in this core was shown in Proposition 1.

Specifically, we begin by showing that the strictly IC allocations in the core of unrestricted mechanisms satisfy equal treatment, and that they converge, as the economy is replicated enough times, to the set of IC ex-post Walrasian allocations. As we have been doing in previous ones, incentive constraints are dropped later in this subsection.

Proposition 5 Consider independent replicas, and suppose that, in every ex-post state, the core convergence theorem holds. ${ }^{13}$

- (i) The strictly IC allocations in the core of unrestricted mechanisms satisfy the equal treatment property.

- (ii) If the economy is sufficiently replicated, $x$ is a strictly IC allocation rule in the economy $\mathcal{E}$ and its replica $x^{m}$ is in the core of unrestricted mechanisms in $\mathcal{E}^{m}$ for every $m$, then $x$ must be an ex-post Walrasian allocation rule.

\footnotetext{
${ }^{13}$ Strictly speaking, we cannot use the theorem in Debreu and Scarf (1963): their consumption set is the non-negative orthant of the $L$-dimensional Euclidean space, while we allow negative consumption in the numeraire. This difference does not affect the validity of the ex-post core convergence result, though.
} 
Proof: (i) First, we show that for every $m \geq 2$, the equal treatment property holds for the core of unrestricted mechanisms under strict IC. Suppose not. That is, for some $i$, some $j, j^{\prime}$, and some $t_{N}, x_{(i, j)}^{m}\left(\bar{t}_{m}\right) \neq x_{\left(i, j^{\prime}\right)}^{m}\left(\bar{t}_{m}\right)$, where $\bar{t}_{m}:=\left(t_{N}, t_{N}, \ldots, t_{N}\right)$. We shall show that there exists a random blocking plan that improves upon $\left(x_{(i, j)}^{m}\right)_{(i, j) \in N \times I_{m}}$.

By the ex-post equal treatment property, there exists a coalition $S \subset$ $N \times I_{m}$ and a feasible plan $y^{S}$, such that $u_{i}\left(y_{i}^{S}\left(\bar{t}_{m}\right), t_{N}\right)>u_{i}\left(x_{i}\left(\bar{t}_{m}\right), t_{N}\right)$ for all $i \in S$. Consider another allocation rule $x^{\prime}$ arbitrarily close to $x$, also strictly IC, satisfying that $x_{i}=x_{i}^{\prime}$ for all $i \notin S$.

Let us consider the following blocking plan $\mu$ for $N \times I_{m}: \mu\left(S, y^{S}\left(\bar{t}_{m}\right), \bar{t}_{m}\right)=$ $\varepsilon>0, \mu\left(N \times I_{m}, x^{\prime}\left(\bar{t}_{m}\right), \bar{t}_{m}\right)=1-\varepsilon>0$ and $\mu$ assigns zero for any other state.

Since $x^{\prime}$ satisfies IC, we can see that both conditions $\left(^{*}\right)$ and $\left(^{* *}\right)$ of Section 2 are satisfied for any $i \notin S$. For $i \in S$, conditions $\left(^{*}\right)$ are clearly satisfied. For the conditions $(* *)-\mathrm{IC}-$, one has two cases:

Case 1: Agent $i$ 's true type is $t_{i}$ and he reports $t_{i}^{\prime}$. Then, the LHS of $(* *)$ is, because of the state probability, approximately proportional to $\varepsilon\left[u_{i}\left(y_{i}^{S}\left(\bar{t}_{m}\right), t_{N}\right)-u_{i}\left(x_{i}^{m}\left(\bar{t}_{m}\right), t_{N}\right)\right]>0$, while the RHS is 0.

Case 2: Agent $i$ 's true type is $t_{i}^{\prime}$ and he reports $t_{i}$. Let $t^{\prime}:=\left(t_{i}^{\prime}, t_{N \backslash\{i\}}\right)$. Then, the LHS of (**) is 0 , whereas the RHS is proportional to $\varepsilon\left[u_{i}\left(y_{i}^{S}\left(\bar{t}_{m}\right), t^{\prime}\right)\right.$ $\left.-u_{i}\left(x_{i}\left(t^{\prime}\right), t^{\prime}\right)\right]+(1-\varepsilon)\left[u_{i}\left(x_{i}^{\prime}\left(\bar{t}_{m}\right), t^{\prime}\right)-u_{i}\left(x_{i}^{m}\left(t^{\prime}\right), t^{\prime}\right)\right]$.

Since $u_{i}\left(x_{i}^{\prime}\left(\bar{t}_{m}\right), t^{\prime}\right)-u_{i}\left(x_{i}^{m}\left(t^{\prime}\right), t^{\prime}\right)<0$ by strict IC, by taking $\varepsilon$ small enough, we can obtain that the RHS is negative. Hence the equal treatment property holds.

(ii) Next we show that if $x$ is a strictly IC allocation rule in the economy $\mathcal{E}$ and its replica $x^{m}$ is in the core of unrestricted mechanisms in $\mathcal{E}^{m}$ for every $m, x$ must be an ex-post Walrasian allocation rule. Suppose not. That is, let $x$ be a strictly IC allocation rule whose replica is in this core of every replicated economy, but suppose that $x\left(t_{N}\right)$ is not a Walrasian allocation for some $t_{N}$.

By the ex-post core convergence theorem, there exists $m$ such that in the $m$-th replication of the ex-post economy in $t_{N}$, there exists a coalition $S$ and a feasible plan $y^{S} \in \mathcal{A}_{S}, S \subseteq N \times I_{m}$, such that $u_{i}\left(y_{i}^{S}\left(\bar{t}_{m}\right), t_{N}\right)>u_{i}\left(x_{i}\left(t_{N}\right), t_{N}\right)$ for all $i \in S$, where $\bar{t}_{m}=\left(t_{N}, t_{N}, \ldots, t_{N}\right)$. Consider another allocation rule $x^{\prime}$ arbitrarily close to $x$, also strictly IC, satisfying that $x_{i}=x_{i}^{\prime}$ for all $i \notin S$. By considering a mechanism similar to the one used above, we can say, using the same arguments, that there exists a randomized mediated blocking for $x^{m}$. 
Remark: The proof of Proposition 5 does not use the assumption that the set of IC ex-post Walrasian allocations is non-empty. In its absence, the proposition implies the non-existence of allocations in the core of unrestricted mechanisms in sufficiently large replicas.

To illustrate the shrinking of the core of unrestricted mechanisms, we shall consider again the economy in the proof of Claim 3.1. Indeed, we show now that $\tilde{x}$, which was shown to be in the core when only measurable blocking mechanisms were considered, is blocked by a non-measurable random blocking plan.

Example 1 Consider the economy in the proof of Proposition 3. First, note that the second replication of $\tilde{x}$ is not in the ex-post core. For instance, it is blocked by coalition $S=\{(2,1),(1,2),(2,2)\}$ in state $(s, s)$ (this notation means that for each of the two replicas the type of individual 1 is s). We denote an ex-post blocking allocation bundle for $S$ as $y^{S}(s, s)$.

For coalition $S^{\prime}=\{(1,1),(2,1)\}$, we define an allocation bundle $y^{S^{\prime}}(s, s)$ as follows: $y_{(1,1)}^{S^{\prime}}(s, s)=(1,1.56)$ and $y_{(2,1)}^{S^{\prime}}(s, s)=(2,0.44)$, which can be obtained from $\tilde{x}(s)$ by an additional transfer of 0.01 units of commodity 2 from individual $(2,1)$ to individual $(1,1)$.

Then, we consider the following blocking plan $\mu: \mu\left(S, y^{S},(s, s)\right)=1-\varepsilon(\varepsilon$ being a very small positive number $), \mu\left(S^{\prime}, y^{S^{\prime}},(s, s)\right)=\varepsilon$, and $\mu\left(T, y^{T}, r\right)=0$ for all coalitions $T$ and states $r \neq(s, s)$.

Then, the blocking plan $\mu$ makes all individuals involved better off. The ex-post objection "almost works" for coalition $S$, except that they do not have the information relevant to agent (2,1). This is the reason to bring agent $(1,1)$ into the blocking plan. Indeed, choosing $\varepsilon$ sufficiently small, individual $(2,1)$ is better off. Because agent $(1,1)$ only participates in the blocking plan with probability $\varepsilon$, in the event he receives the phone call from the blocking mediator, he accepts the plan because he also improves. Effectively, in this event he "sells" his information to agent $(2,1)$ for 0.01 additional units of the numeraire. Recall that each agent believes that the status quo is still available in the event he is not part of the blocking plan, and under these beliefs, types are reported truthfully to the blocking plan.

Finally, it is easy to choose $y^{S}(s, s)$ appropriately to ensure that the blocking plan satisfies IC. Note in particular how this can be done so that the other types of agents $(1,1)$ and $(1,2)$ do not have an incentive to misrepresent their 
types or accept the blocking plan. They optimally choose to truthfully stay out of it (equations $\left(^{*}\right)$ and (**) applied to them).

If one drops the incentive constraints, one obtains the following parallel result:

Proposition 6 Consider independent replicas, and suppose that incentive constraints are dropped, and that, in every ex-post state, the core convergence theorem holds.

- (i) The allocations in the core of unrestricted mechanisms satisfy the equal treatment property.

- (ii) If the economy is sufficiently replicated, $x$ is an allocation rule in the economy $\mathcal{E}$ and its replica $x^{m}$ is in the core of unrestricted mechanisms in $\mathcal{E}^{m}$ for every $m$, then $x$ must be an ex-post Walrasian allocation rule.

Proof: The arguments in the proof of Proposition 5 do not rely on $x$ being IC and $x^{\prime}$ being strictly IC. All that matters is the equal treatment property of the ex-post core and the ex-post core convergence theorem. After one realizes this, the proof is simpler since one does not need to check conditions $(* *)$.

\section{Ex-Post Replica Economies}

Different from the independent replica process studied so far, Serrano, Vohra and Volij (2001) propose ex-post replicas. Ex-post replicas result in information becoming non-exclusive already in the second replica, which in turn makes incentive constraints redundant. This is a different sense of defining a negligible size of agents from the informational point of view.

Formally, the only difference of this replica process with respect to the one defined in the previous section is the following. Let $T=\prod_{i=1}^{n} T_{i}$ be the set of ex-post states in the basic economy. This will remain the set of ex-post states even after replication. For each $(i, j) \in N \times I_{m}, t_{(i, j)}=t_{\left(i, j^{\prime}\right)}=t_{i}$, and $q_{m}\left(t_{-i} \mid t_{i}\right)=q\left(t_{-i} \mid t_{i}\right)$.

For this replica process, Serrano, Vohra and Volij (2001) exhibited a robust failure of the core convergence theorem for core notions that did not 
model the information transmission by means of equilibrium blocking. However, the main result of this section is the following:

Proposition 7 Consider ex-post replicas, and suppose that incentive constraints are either present or dropped, and that, in every ex-post state, the core convergence theorem holds.

- (i) The allocations in the core of single-coalition deterministic mechanisms satisfy the equal treatment property.

- (ii) If the economy is sufficiently replicated, $x$ is an allocation rule in the economy $\mathcal{E}$ and its replica $x^{m}$ is in the core of single-coalition deterministic mechanisms in $\mathcal{E}^{m}$ for every $m$, then $x$ must be an ex-post Walrasian allocation rule.

Proof: First, as observed above, already in the second replica, the economy is one of non-exclusive information, and therefore, even if incentive constraints were relevant in the basic economy, they can be dropped from the second replica onwards.

Now consider an allocation $x$ whose replica $x^{m}$ is in the core of singlecoalition deterministic mechanisms in every ex-post replicated economy. When incentive constraints are dropped, this core is identical to the fine core (Dutta and Vohra (2005), Serrano and Vohra (2007)). Moreover, in quasilinear economies, the ex-post core is a subset of the fine core (Dutta and Vohra (2005)), and adapting an argument for the continuum (Einy, Moreno and Shitowitz (2000b)) to large enough replica economies, the fine core is a subset of the ex-post core. Therefore, by combining these statements, we obtain that allocations in the core of single-coalition deterministic mechanisms satisfy the equal treatment property and converge to the set of ex-post Walrasian allocations.

Remark: Putting together Propositions 7 and 2, we find out that, when incentive constraints are dropped, in large enough quasilinear economies, the core of single-coalition deterministic mechanisms is a subset of the ex-post core, itself a subset of the core of unrestricted mechanisms. Therefore, all three converge to the same set in large economies. This is not true if incentive constraints remain relevant. 
Remark: Making use of the same steps as in the proof of Proposition 7 and of Aumann's (1964) core equivalence theorem for the economy with an atomless continuum of agents, one can easily establish the same equivalence theorem for the core of single-coalition deterministic mechanisms. Recall that in this section it is very important that the set of states of the world is finite.

Therefore, a convergence/equivalence theorem has been established for this case. Interestingly, when the set of states of nature is not replicated with the economy, there is no reason to resort to the full force of random blocking plans to obtain it. It suffices to apply the equilibrium logic to blocking plans, even for those involving only a deterministic allocation rule coming from a single coalition.

\section{Signal-Based Replica Economies}

We consider now a different replica process, which we call signal-based replicas. This was first considered in McLean and Postlewaite (2002). Agents' utility functions will depend on an underlying but unobserved state of nature $\theta$, and each agent will receive a private signal that is correlated with the state of nature. A replication of this initial economy consists of a set of agents whose utility functions and endowments are the same as those in the underlying initial economy, but whose private signals across cohorts are independent conditional on $\theta$. As the number of replicas increases, each agent becomes small in the economy in terms of endowment, and each agent is also "informationally small": the conditional distribution on the state of nature does not vary much in that agent's signal if other agents' signals are known. Note that no agent's information is redundant in this replication process: regardless of the number of replications, each agent still has information that cannot be inferred from the aggregate information of other agents.

\subsection{Notation and definitions}

Since the model employed in these replicas is slightly different from the rest of the paper, we begin with a few preliminaries. Let $\Theta=\left\{\theta_{1}, \ldots, \theta_{m}\right\}$ denote the (finite) state space and let $T_{1}, \ldots, T_{n}$ be finite sets, where $T_{i}$ represents the set of possible signals that agent $i \in N$ might receive. In this model, nature chooses an element $\theta \in \Theta$. The state of nature is unobservable but 
each agent $i$ receives a "signal" that is correlated with nature's choice of $\theta$. We denote the probability distribution on $\Theta \times T$ as $P$.

The consumption set of each agent $X_{i}$ is $R_{+}^{l-1} \times R$ and for each $\theta \in \Theta$, $\omega_{i} \in X_{i}$ denotes the (state independent) initial endowment of agent $i$ in state $\theta \in \Theta$. The preferences of agent $i$ are given by a utility function $u_{i}: R_{+}^{l-1} \times R \times \Theta \rightarrow R$ where $u_{i}\left(x_{i}, \theta\right)=v_{i}^{-l}\left(x_{i}^{-l}, \theta\right)+x_{i}^{l}$ is the utility function of agent $i$ in state $\theta$. We note that in this model agents' utility functions do not depend on $T_{N}$. The collection $e=\left(\left\{u_{i}, X_{i}, \omega_{i}, T_{i}\right\}_{i \in N}, \Theta, P\right)$ will be called a private information economy. It will be assumed that the data defining the private information economy is common knowledge among the agents. A private information economy allocation $x=\left(x_{1}, \ldots, x_{n}\right)$ for the private information economy is a collection of functions $x_{i}: T_{N} \rightarrow \mathcal{A}_{N}$.

For each state $\theta_{k} \in \Theta$, the collection $\left\{u_{i}\left(\cdot, \theta_{k}\right), \omega_{i}\right\}_{i \in N}$ defines an associated (complete information) economy. Let $\left(z_{i}^{*}\left(\theta_{k}\right)\right)_{i \in N}$ denote a Walrasian equilibrium allocation in state $\theta_{k}$.

Let us define a sequence of signal-based replica economies. Recall that $I_{m}=\{1,2, \ldots, m\}$. Given the collection $\left\{\omega_{i}, u_{i}\right\}_{i \in N}$ and $m$, let $\left\{\omega_{(i, j)}, u_{(i, j)}\right\}_{(i, j) \in N \times I_{m}}$ denote the $m$ replication of $\left\{\omega_{i}, u_{i}\right\}_{i \in N}$ satisfying:

(1) $\omega_{(i, j)}=\omega_{i}$ for all $i \in N$ and all $j \in I_{m}$,

(2) $u_{(i, j)}(x, \theta)=u_{i}(x, \theta)$ for all $x \in R_{+}^{l-1} \times R, i \in N$ and $j \in I_{m}$.

For any positive integer $m$, let $T^{m}=T \times \cdots \times T$ denote the $m$-fold Cartesian product and let $t^{m}$ denote a generic element of $T^{m}$. Let $P_{m}$ be a probability distribution on $\Theta \times T^{m}$. Then $e^{m}=\left(\left\{u_{(i, j)}, X_{(i, j)}, \omega_{(i, j)}, T_{(i, j)}\right\}_{(i, j) \in N \times I_{m}}, \Theta, P_{m}\right)$ is a private information economy with $n \times m$ agents.

A sequence of replica economies $\left\{\left(\left\{u_{(i, j)}, X_{(i, j)}, \omega_{(i, j)}, T_{(i, j)}\right\}_{(i, j) \in N \times I_{m}}, \Theta, P_{m}\right)\right\}_{m=1}^{\infty}$ is a conditionally independent sequence if

(a) For each $m$, each $j \in I_{m}$, and each $\left(\theta, t_{1}, \ldots, t_{n}\right) \in \Theta \times T$,

$$
P\left(\theta, t_{(1, j)}^{m}, t_{(2, j)}^{m}, \ldots, t_{(n, j)}^{m}\right)=P\left(\theta, t_{1}, t_{2}, \ldots, t_{n}\right) ;
$$

(b) for each $m$ and each $\theta$, the probability distributions over

$$
\left(T_{(1,1)}^{m}, T_{(2,1)}^{m}, \ldots, T_{(n, 1)}^{m}\right), \ldots,\left(T_{(1, m)}^{m}, T_{(2, m)}^{m}, \ldots, T_{(n, m)}^{m}\right)
$$

are independent given $\theta$;

(c) for every $\theta, \theta^{\prime}$ with $\theta \neq \theta^{\prime}$, there exists a $t_{N} \in T_{N}$ such that $P\left(t_{N}\right.$ $\theta) \neq P\left(t_{N} \mid \theta^{\prime}\right)$.

Thus a conditionally independent sequence is a sequence of private information economies with $n \times m$ agents containing $m$ "copies" of each agent 
$i \in N$. It follows that, for large enough $m$ and for almost every signal profile in the replica economy, one could assign a probability to a state that is arbitrarily close to 1 .

\subsection{Convergence Result}

In this subsection we provide a convergence result for this model. In each state $\theta_{k}$, we make the following (strongly) ex-post core convergence assumption: ${ }^{14}$

Assumption 1: For every $\varepsilon>0$, there exists an integer $\hat{m}$ such that for all $m>\hat{m}$, if a feasible allocation in the $m$-fold economy, $x^{m} \in \mathcal{A}_{N \times I_{m}}$ satisfies $u_{i}\left(x_{i}^{m}, \theta_{k}\right)-u_{i}\left(z_{i}^{*}\left(\theta_{k}\right), \theta_{k}\right)>\varepsilon$, then there exist a coalition $S$ and $y^{S} \in \mathcal{A}_{S}$ such that $\sum_{j \in S} u_{j}\left(y_{j}^{S}, \theta_{k}\right)>\sum_{j \in S} u_{j}\left(x_{j}^{m}, \theta_{k}\right)+\frac{\varepsilon}{2}$.

This assumption implies the following:

Assumption 1': In each state $\theta_{k}$, for all $\varepsilon>0$ there exists an integer $\hat{m}$ such that for all $m>\hat{m}$, if a feasible allocation in the $m$-fold economy, $x^{m} \in \mathcal{A}_{N \times I_{m}}$ satisfies

$$
\#\left\{j \in I_{m} \mid u_{i}\left(x_{(i, j)}^{m}, \theta_{k}\right)-u_{i}\left(z_{i}^{*}\left(\theta_{k}\right), \theta_{k}\right)>\varepsilon\right\}>\varepsilon m
$$

for some $i \in N$, then there exist $S \subset N \times I_{m}$ and $y^{S} \in \mathcal{A}_{S}$ such that $\sum_{j \in S} u_{j}\left(y_{j}^{S}, \theta_{k}\right)>\sum_{j \in S} u_{j}\left(x_{j}^{m}, \theta_{k}\right)+\frac{\varepsilon}{2} \times(\varepsilon m)$.

We show that when $m$ is large, and except for very unlikely signal profiles, allocations in the strict IC core of unrestricted mechanisms for the $m$-fold private information economy will give most agents utility that is close to that of some Walrasian equilibrium allocation in some state $\theta_{k}$. The following proposition formalizes this statement. It says that for large enough replicas the joint probability of a specific informational event is arbitrarily close to 1. The informational event in question consists of those signal profiles for which at any core allocation the ex-post utility of almost all agents is arbitrarily close to that obtained at a strongly ex-post Walrasian allocation in some state:

\footnotetext{
${ }^{14}$ Strongly ex-post refers to the timing after the state of the world would be observed (something that never happens in this model), while ex-post would refer to the signal profile being commonly known by all agents, something that could happen if they chose to pool their information.
} 
Proposition 8 Consider conditionally independent signal-based replicas of a quasilinear economy in our admissible class. For every $\varepsilon>0$, there exists an $\hat{m}>0$ such that for all $m>\hat{m}$, every strictly IC allocation in the core of unrestricted mechanisms $x^{m}$ of $m$-replicated economies satisfies

$$
\begin{aligned}
\operatorname{Pr}\left\{t^{m} \in T^{m}\right. & \mid \exists k \text { s.t. } \\
& \#\left\{j \in I_{m}|| u_{i}\left(x_{(i, j)}^{m}\left(t^{m}\right), \theta_{k}\right)-u_{i}\left(z_{i}^{*}\left(\theta_{k}\right), \theta_{k}\right) \mid<\varepsilon\right\} \geq(1-\varepsilon) m \\
& \text { for all } i \in N\} \geq 1-\varepsilon,
\end{aligned}
$$

where $\left(z_{i}^{*}\left(\theta_{k}\right)\right)_{i \in N}$ is a Walrasian equilibrium allocation in $\theta_{k}$.

Proof: Let $m_{1}$ be the number satisfying Assumption 1'. That is, in each state $\theta_{k}$, for all $m>m_{1}$, if a feasible allocation in $m$-fold economy, $x^{m} \in$ $\mathcal{A}_{N \times I_{m}}$ satisfies

$$
\#\left\{j \in I_{m} \mid u_{i}\left(x_{(i, j)}^{m}, \theta_{k}\right)-u_{i}\left(z_{i}^{*}\left(\theta_{k}\right), \theta_{k}\right)>\varepsilon\right\}>\varepsilon m
$$

for some $i \in N$, then there exist $S \subset N \times I_{m}$ and $y^{S} \in \mathcal{A}_{S}$ such that $\sum_{j \in S} u_{j}\left(y_{j}^{S}, \theta_{k}\right)>\sum_{j \in S} u_{j}\left(x_{j}^{m}, \theta_{k}\right)+\frac{\varepsilon}{2} \times(\varepsilon m)$.

Let $\eta:=\min \left\{\varepsilon, \frac{\varepsilon^{2}}{\varepsilon^{2}+2 M n}\right\}$, where $M:=\max _{\theta} \max _{i} v_{i}^{-l}\left(\sum_{j=1}^{n} \omega_{j}^{-l}, \theta\right)+$ $\sum_{j=1}^{n} \omega_{j}^{l}$. We note that $\eta$ satisfies $(1-\eta) \cdot \frac{\varepsilon^{2}}{2}>\eta \cdot M \cdot n$.

Applying the argument in Gul and Postlewaite (1992, p.1290), it follows that for every $\eta>0$ there exists an integer $m_{2}$ satisfying the following: for all $m>m_{2}$,

$$
\operatorname{Pr}\left\{t^{m} \in T^{m} \mid \text { for some } k, \operatorname{Pr}\left(\theta=\theta_{k} \mid t^{m}\right) \geq 1-\eta\right\} \geq 1-\eta .
$$

Let $\hat{m}:=\max \left\{m_{1}, m_{2}\right\}$.

Suppose that $m>\hat{m}$. Let $B_{k}^{m}:=\left\{t^{m} \in T^{m} \mid \operatorname{Pr}\left(\theta=\theta_{k} \mid t^{m}\right) \geq\right.$ $1-\eta\}$. We show that for every $t^{m} \in B_{k}^{m}$, every strictly IC allocation in the core of unrestricted mechanisms $x^{m}$ satisfies $\#\left\{j \in I_{m}|| u_{i}\left(x_{(i, j)}^{m}\left(t^{m}\right), \theta_{k}\right)-\right.$ $\left.u_{i}\left(z_{i}^{*}\left(\theta_{k}\right), \theta_{k}\right) \mid<\varepsilon\right\} \geq(1-\varepsilon) m$ for all $i \in N$.

Suppose not. Then \# $\left\{j \in I_{m} \mid u_{i}\left(x_{(i, j)}^{m}\left(t^{m}\right), \theta_{k}\right)-u_{i}\left(z_{i}^{*}\left(\theta_{k}\right), \theta_{k}\right)>\varepsilon\right\}>\varepsilon m$ for some $i \in N$. By considering a random mechanism similar to the one in the proof of Proposition 5, it would be enough to show that there exists an ex-post objection in $t^{m}$.

By using assumption 1', there exists a coalition $S \subset N \times I_{m}$ and $y^{S} \in \mathcal{A}_{S}$ such that $\sum_{j \in S} u_{j}\left(y_{j}^{S}, \theta_{k}\right)>\sum_{j \in S} u_{j}\left(x_{j}^{m}\left(t^{m}\right), \theta_{k}\right)+\frac{\varepsilon}{2} \times(\varepsilon m)$. Then 


$$
\begin{aligned}
\sum_{j \in S} \sum_{\theta \in \Theta} u_{j}\left(y_{j}^{S}, \theta\right) P\left(\theta \mid t^{m}\right) & >(1-\eta) \sum_{j \in S} u_{j}\left(y_{j}^{S}, \theta_{k}\right) \\
& >(1-\eta) \sum_{j \in S} u_{j}\left(x_{j}^{m}\left(t^{m}\right), \theta_{k}\right)+(1-\eta) \cdot \frac{\varepsilon^{2}}{2} m \\
& \geq(1-\eta) \sum_{j \in S} u_{j}\left(x_{j}^{m}\left(t^{m}\right), \theta_{k}\right)+\eta \cdot M n \cdot m \\
& \left.>\sum_{j \in S} \sum_{\theta \in \Theta} u_{j}\left(x_{j}^{m}\left(t^{m}\right), \theta\right) P\left(\theta \mid t^{m}\right) \text { (because }|S| \leq n \times m\right) .
\end{aligned}
$$

Since agents' utilities are quasilinear, this inequality implies that $y^{S}$ is an ex-post objection to $x^{m}$ in $t^{m}$.

Remark: Unlike Proposition 5, this proof relies on quasilinearity.

Remark: McLean and Postlewaite (2005) establish a positive convergence result for the ex-ante IC core under strongly conditionally independent sequences, which means that the distributions over each $T_{j}$ are also independent given $\theta$ in an original economy (compare with part (b) in the definition above).

Once again, noting that the core shrinking argument in the proof of Proposition 8 does not rely on the use of incentive constraints, one realizes that these can be dropped. The relevant framework now would be one in which signals are publicly verifiable, but not the state of nature, which remains unobservable. Then, one can state our final result, whose proof can be omitted:

Proposition 9 Consider conditionally independent signal-based replicas of a quasilinear economy in our admissible class in which incentive constraints are dropped. For every $\varepsilon>0$, there exists an $\hat{m}>0$ such that for all $m>\hat{m}$, every allocation in the core of unrestricted mechanisms $x^{m}$ of m-replicated economies satisfies

$$
\begin{aligned}
\operatorname{Pr}\left\{t^{m} \in T^{m}\right. & \mid \exists k \text { s.t. } \\
& \#\left\{j \in I_{m}|| u_{i}\left(x_{(i, j)}^{m}\left(t^{m}\right), \theta_{k}\right)-u_{i}\left(z_{i}^{*}\left(\theta_{k}\right), \theta_{k}\right) \mid<\varepsilon\right\} \geq(1-\varepsilon) m \\
& \text { for all } i \in N\} \geq 1-\varepsilon,
\end{aligned}
$$


where $\left(z_{i}^{*}\left(\theta_{k}\right)\right)_{i \in N}$ is a Walrasian equilibrium allocation in $\theta_{k}$.

\section{Concluding Remarks}

This paper has studied equilibrium blocking in large quasilinear economies. Results vary as a function of the class of communication mechanisms employed by coalitions and also of the replica process used. The main factor that accounts for the difference in results is the amount of information transmission that one permits each coalition to use. If random coalition formation is possible and information can be used, within a blocking plan, from coalition to coalition in the plan, a positive convergence result was obtained to the set of incentive compatible ex-post Walrasian allocations, whenever this is nonempty. But such a positive convergence result is prevented in independent replicas if blocking communication mechanisms are restricted to being either measurable or deterministic. On the other hand, in ex-post replicas there is no need to go beyond deterministic mechanisms to obtain a convergence result. In signal-based replicas, where our results are weaker, a probabilistic convergence result was obtained for the core of unrestricted mechanisms.

We close by reiterating a very important observation. We note that, while we have been using the incentive constraints to motivate our analysis -in part, because the approach is rooted in mechanism design-, all our results extend to the case in which such constraints are not imposed. It then follows that the relevance of the results in the current paper is enhanced substantially, as existence of ex-post Walrasian allocations is readily obtained. ${ }^{15}$

\footnotetext{
${ }^{15}$ See also de Clippel (2007) for a different core convergence result when incentive constraints are ignored.
} 


\section{References}

Allen, B., (1981). "Generic Existence of Completely Revealing Equilibria for Economies with Uncertainty when Prices Convey Information," Econometrica 49, 1173-1199.

Allen, B., Yannelis, N. C., (2001). "Differential Information Economies: Introduction," Economic Theory 18, 263-273.

Anderson, R. M. (2008). "Core Convergence," In S. Durlauff and L. Blume (eds.): The New Palgrave Dictionary of Economics, 2nd edition, McMillan, London.

Aumann, R. J., (1964). "Markets with a Continuum of Traders," Econometrica 32, 39-50.

Blouin, M., Serrano, R. (2001). "A Decentralized Market with Common Values Uncertainty: Non-Steady States," Review of Economic Studies, 68, 323-346.

de Clippel, G., (2007). "The Type-Agent Core of Exchange Economies with Asymmetric Information," Journal of Economic Theory 135, 144-158.

de Clippel, G., Minelli, E., (2005). "Two Remarks on the Inner Core," Games and Economic Behavior 50, 143-154.

Debreu, G., Scarf, H., (1963). "A Limit Theorem on the Core of an Economy," International Economic Review 4, 235-246.

Dubey, P., Geanakoplos, J., Shubik, M. (1987). "The Revelation of Information in Strategic Market Games: A Critique of Rational Expectations Equilibrium," Journal of Mathematical Economics, 6, 105-137.

Dutta, B., Vohra, R., (2005). "Incomplete Information, Credibility and the Core," Mathematical Social Sciences 50, 148-165.

Einy, E., Moreno, D., Shitovitz, B., (2000a). "Rational Expectations Equilibria and the Ex-Post Core of an Economy with Asymmetric Information," Journal of Mathematical Economics 34, 527-535.

Einy, E., Moreno, D., Shitovitz, B., (2000b). "On the Core of an Economy with Differential Information," Journal of Economic Theory 94, 262-270.

Forges, F., Minelli, E. (1997). "Self-Fulfilling Mechanisms and Rational Expectations," Journal of Economic Theory, 75, 388-406.

Forges, F., Heifetz, A., Minelli, E., (2001). "Incentive Compatible Core and Competitive Equilibria in Differential Information Economies", Economic Theory 18, 349-365.

Forges, F., Mertens, J.-F., Vohra, R., (2002). "The Ex Ante Incentive Compatible Core in the Absence of Wealth Effects," Econometrica 70, 1865- 
1892.

Forges, F., Minelli, E., Vohra, R., (2002). "Incentives and the Core of an Exchange Economy: a Survey," Journal of Mathematical Economics 38, $1-41$.

Foster, D. F., Viswanathan, S. (1996). "Strategic Trading When Agents Forecast the Forecast of Others," Journal of Finance 51, 1437-1478.

Gottardi, P., Serrano, R. (2005). "Market Power and Information Revelation in Dynamic Trading," Journal of the European Economic Association 3, 1279-1317.

Gul, F., Postlewaite, A., (1992). "Asymptotic Efficiency in Large Exchange Economies with Asymmetric Information," Econometrica 60, 1273-1292.

Holden, C. W., Subrahmanyam, A. (1992). "Long-Lived Private Information and Imperfect Competition," Journal of Finance, 47, 247-270.

Kreps, D., (1977). "A Note on Fulfilled Expectations Equilibria," Journal of Economic Theory 14, 32-43.

Kyle, A. (1985). "Continuous Auctions and Insider Trading," Econometrica 53, $1315-1335$.

Laffont, J.-J., (1985). "On the Welfare Analysis of Rational Expectations Equilibria with Asymmetric Information," Econometrica 53, 1-29.

McLean, R. P., Postlewaite, A., (2002). "Informational Size and Incentive Compatibility," Econometrica 70, 2421-2453.

McLean, R., Postlewaite, A., (2005). "Core Convergence with Asymmetric Information," Games and Economic Behavior 50, 58-78.

Milgrom, P. (1981). "Rational Expectations, Information Acquisition, and Competitive Bidding," Econometrica 49, 921-943.

Myerson, R. B., (1991). Game Theory: Analysis of Conflict, Harvard University Press, Cambridge, MA.

Myerson, R. B., (2007). "Virtual Utility and the Core for Games with Incomplete Information," Journal of Economic Theory 136, 260-285.

Pesendorfer, W., Swinkels, J. (1997). "The Loser's Curse and Information Aggregation in Common Values Auctions," Econometrica 65, 12471281.

Peters, M., Severinov, S. (2008). "An Ascending Double Auction," Economic Theory 37, 281-306.

Postlewaite, A., Schmeidler D., (1986). "Implementation in Differential Information Economies," Journal of Economic Theory 39, 14-33.

Qin, C.-Z., (1993). "The Inner Core and the Strictly Inhibitive Set," Journal of Economic Theory 59, 431-444. 
Radner, R., (1979). "Rational Expectations Equilibrium: Generic Existence and the Information Revealed by Prices," Econometrica 47, 655-678.

Reny, P., Perry, M. (2006). "Toward a Strategic Foundation for Rational Expectations Equilibrium," Econometrica 74, 1231-1269.

Serrano, R., Vohra, R., (2007). "Information Transmission in Coalitional Voting Games," Journal of Economic Theory 134, 117-137.

Serrano, R., Vohra, R., Volij, O., (2001). "On the Failure of Core Convergence in Economies with Asymmetric Information," Econometrica 69, $1685-1696$.

Shapley, L., Shubik, M. (1977). "Trade Using One Commodity as Means of Payment," Journal of Political Economy, 85, 937-968.

Vohra, R., (1999). "Incomplete Information, Incentive Compatibility and the Core," Journal of Economic Theory 86, 123-147.

Wilson, R., (1978). "Information, Efficiency and the Core of an Economy," Econometrica 46, 807-816.

Wolinsky, A. (1990). "Information Revelation in a Market with Pairwise Meetings," Econometrica 58, 1-23. 